$\$$ Research Square

\title{
An invasive shrub Lantana camara L. alters the flora and soils in tropical dry deciduous forests of Central India
}

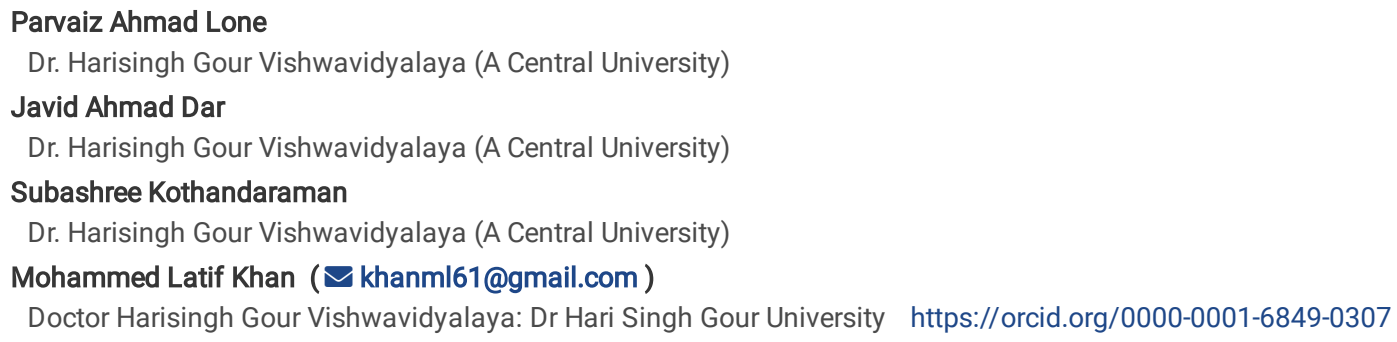

\section{Research Article}

Keywords: Impact, stand structure, composition, diversity, soils, Lantana camara, tropical forests, Central India

Posted Date: February 17th, 2021

DOI: https://doi.org/10.21203/rs.3.rs-223195/v1

License: (c) (i) This work is licensed under a Creative Commons Attribution 4.0 International License. Read Full License 


\section{Abstract}

Background and Aims

Lantana camara is a highly noxious invasive weed species of global concern. However, its impacts on floristic and soil properties in tropical dry deciduous forests are elusive and fragmented. We aimed to assess the changes in the flora and soil properties following the invasion by Lantana camara in Central Indian forest ecosystems.

Methods

Three study sites were selected and each site was further divided into two subsites: Lantana-invaded (LI) and uninvaded (UI). In total, 60 plots of 0.25 ha each (10 plots in each subsite) were laid randomly. Within each plot floristic structure, composition, diversity and soil SOC, STN, M\%, pH and bulk density were assessed.

Results

Lantana-invaded sites showed a significant decrease in density (D), basal area (BA), species richness (SR) and evenness (E) of seedlings ( $<3 \mathrm{~cm}$ diameter at breast height $(\mathrm{DBH})$ ), juveniles (> 3-9.9cm DBH), and herbs. In LI sites, a reduction of 57 and $25 \%$ has been observed in lower DBH class of trees $(<3 \mathrm{~cm}$ and > 3-9.9cm). In all LI sites, a significant increase of soil organic carbon (SOC), soil total nitrogen (STN) and soil moisture (M\%) and a significant decrease of pH and bulk density (BD) were recorded.

Conclusions

Lantana may greatly impact the vegetation and soil properties and, successively, these strong changes increase its invasive potential and ability to replace native species by averting their natural regeneration potential. Therefore, a proper management strategy of this noxious weed is imperative to prevent its further expansion and future problems.

\section{Introduction}

Biological invasions are a major threat to global biodiversity and have greater ecological impacts in a wide range of ecosystems worldwide (Seebens et al. 2017; Lazzaro et al. 2020). Invasive species are the second biggest threat to biodiversity loss after habitat fragmentation (Simberloff et al. 2013; Ahmad et al. 2019) as they pose detrimental impacts on the native local biodiversity, ecosystems, economy and human health (D'Antonio and Vitousek 1992; Levine et al., 2003; Hejda and Pysek 2006; Hejda et al. 2009; Ramírez-Albores et al. 2019). They are found in almost all terrestrial ecosystems and are important drivers of global change biology (Vitousek, 1994). Invasive species impact ecosystems by altering the fire regimes, geomorphology (Fei et al. 2014; Gaertner et al. 2014) and have substantial impacts on both ecosystem structure and functioning, such as reduction in native species diversity, changes in ecosystem productivity and alteration of soil nutrient pools (Liao et al. 2008; Pysek et al. 2012; Barney et al. 2015).

Invasive species are often perceived as those species that were introduced in a particular location intentionally or unintentionally that penetrates and replaces the prevailing indigenous vegetation of a location (Rejmanek 1995) and are known to have a wide range of ecological impacts on introduced ecosystems such as competitive exclusion of native species, increased water loss, alterations in nutrient dynamics, fire regimes, etc. (Truscott et al 2008). Invasion by alien plant species alters the native community structure and composition, cause local extinctions of resident species, alter several ecological processes, successional rates and trajectories (Vila et al. 2011; Potgieter et al. 2014; Bellard et al. 2016). Invasive plants not only alter the structural and functional diversity of ecosystems, but also change the properties of soils, such as $\mathrm{pH}$, moisture, bulk density, soil organic carbon, total nitrogen, phosphorus and other soil micro-biota (Ehrenfeld, 2003; Dassonville et al., 2008; Sharma and Raghubanshi 2009; Timsina et al. 2011; ). The success of invasive plants is often due to its particular traits such as phenotypic plasticity, short-life spans, pollination by generalists, high fecundity, rapid growth rates, allelopathy, etc. (Rejmanek 1995). Invasive plants can trigger new complex interactions with the existing native species and also with other co-invading species (Hartman and McCarthy 2008). As invasive species drastically alter the structure and functioning of ecosystems, in the changing global climatic warming, the rising rate of plant invasions are regarded as a major threat to the global biodiversity (Hellmann et al. 2008; Catford et al. 2012; Taylor et al. 2012).

Tropical forests are known to have rich species diversity and tropical dry forests are the most endangered and degraded of all ecosystems in the world (Janzen 1988; Cabin et al. 2002). Several invasive species are able to establish viable populations in mature tropical forests and are a cause of concern (Brown et al. 2006). The tropical dry forests have canopy openings, grazing, fires etc., that act as windows for invasive species proliferation (Johnstone 1986; Raghubanshi and Tripathi 2009). There is a growing consensus that invasive plants create 'novel' tropical ecosystems with vegetation transitions (Veldman and Putz 2010). Furthermore, shifts in vegetation transitions are often accompanied by alterations in soil physico-chemical properties due to changes in the timing, duration, quality and quantity of plant-derived organic substrates (Ehrenfeld et al. 2010) that lead to further degradation. The facilitation of invasion by forest degradation is often overlooked, particularly in the tropical forests of developing countries such as India (Mungi et al. 2020).

Despite the numerous studies on the impacts of invasive species, only few have studied their impacts on the vegetation structure, composition, diversity, and soil properties in tropical and temperate forest ecosystems (Sharma and Raghubanshi 2009; Sundaram and Hiremath 2012; Sharma and Raghubanshi 2010; Dobhal et al. 2011; Kumar et al. 2020). Assessing the impacts on the structural and functional diversity is important, because they are influenced by each other, and the changes in vegetation structure, composition and diversity due to invasions would eventually result in alterations in soil properties and viceversa (Vitousek, 1990) 
Alike to many invasive plants, Lantana camara is a vigorously growing shrub that is highly invasive in over 60 countries with about 650 varieties (Global Invasive Species Database 2020). It is widespread throughout the tropical, subtropical and warm temperate regions covering about 13 million hectares (Sharma et al. 2005; Goyal et al. 2018) and a major threat to $44 \%$ of the total Indian forests (Mungi et al. 2020). It has almost spread in all the dry deciduous forests of India (Sharma and Raghubanshi 2006). Most of the past studies have centered on the effects of invasion on the herbaceous and seeding vegetation, and the research on the impacts of invasive species on vegetation composition, diversity and soil properties in tropical dry deciduous forests of India, particularly in Central Indian forests is scare and fragmented. To bridge this gap, the present study has been aimed: 1 ) to examine the impact of $L$. camara on the vegetation structure, composition and diversity of tree saplings, juveniles, adults, herbs and shrubs \& lianas, and 2 ) the impact of $L$. camara on soil properties in tropical dry deciduous forests of Central India.

\section{Materials And Methods Study species}

Lantana camara L. (Verbenaceae) is a woody shrub native to Central and South America and is regarded as one of the ten worst invasive species in the world (Richardson and Rejmanek 2011). It was introduced as an ornamental hedge plant in East India Company Botanical Gardens in Calcutta in 1809, from where it escaped and became invasive (Kohli et al. 2006; Kannan et al. 2013). The plant is profusely branched and grows up to 2-4 meters ( $\mathrm{m}$ ) high in open unshaded sunny environments (Day et al. 2003), and as a liana up to $15 \mathrm{~m}$ when light intensity is low (Lowe et al. 2000). It is shade-tolerant and produces 10,000-12,000 fruits (Kohli et al. 2006) and is very commonly distributed across landscapes in fragmented dry deciduous forests of Central India (Mungi et al. 2020).

\section{Study area description}

The present study was conducted in three forest study sites (Kesli, Deori and Shahgarh ranges) located between $21^{\circ} 17^{\prime}-26^{\circ} 52 \rrbracket \mathrm{N}$ and $78^{\circ} 08 \rrbracket-82^{\circ} 49 \square \mathrm{E}$ in district Sagar of Madhya Pradesh (M.P), Central India (Table 1, Fig. 1). The area is situated in the north central region of M.P. and is covered by Vindhyanchal mountain range at an average height of $420 \mathrm{~m}$ asl. The forest in the area belongs to group $4 \mathrm{~b}$ of the Champion and Seth's classification (Champion and Seth 1968) and the climate is subtropical with hot dry summers (March to mid-June), monsoon season (mid-June to September) and, cool and dry winters (October to February). The area receives an annual average rainfall of $1197.6 \mathrm{~mm}$ of which approximately $90 \%$ takes place during the southwest monsoon. The mean annual minimum and maximum temperatures vary between $11.6-40.7^{\circ} \mathrm{C}$ in January and May, respectively. The major soil types of the area are clay loam, sandy clay loam and sandy loam. The vegetation of the area is characterized by tropical dry deciduous forests, predominated by species of Tectona grandis, Diospyros melanoxylon Butea monosperma and Lagerstroemia parviflora. During the last few decades, these forests have been severely infested by plant invasions, particularly Lantana (Dar et al. 2019) and impacted the physical, chemical and biological aspects of ecosystems (Lone et al. 2019).

Table 1

Study site characteristics of uninvaded (UI) and Lantana-invaded (LI) sites of tropical dry deciduous forest of Sagar, Madhya Pradesh

\begin{tabular}{|c|c|c|c|c|c|c|}
\hline \multirow[t]{2}{*}{ Parameter } & \multicolumn{3}{|l|}{ Uninvaded (UI) } & \multicolumn{3}{|c|}{ Lantana-invaded (LI) } \\
\hline & Site-1 & Site-2 & Site-3 & Site-1 & Site-2 & Site-3 \\
\hline Latitude $\left({ }^{\circ}\right)$ & $23.46-23.47$ & $23.19-23.20$ & $24.39-24.39$ & $23.46-23.47$ & $23.19-23.20$ & $24.39-24.39$ \\
\hline Longitude $\left(^{\circ}\right)$ & 78.77-78.78 & $79.03-79.04$ & $79.23-79.23$ & 78.77-78.78 & $79.03-79.04$ & $79.23-79.23$ \\
\hline Altitude $(\mathrm{m})$ & $536-568$ & $428-476$ & $386-397$ & $535-588$ & $435-460$ & $378-393$ \\
\hline No. of plots & 10 & 10 & 10 & 10 & 10 & 10 \\
\hline Tree density (no. ha ${ }^{-1}$ ) & 528 & 443 & 400 & 343 & 354 & 300 \\
\hline $\begin{array}{l}\text { Tree basal area } \\
\left(\mathrm{m}^{2} \mathrm{ha}^{-1}\right)\end{array}$ & 22.5 & 18.2 & 18.3 & 15.2 & 12.3 & 16.5 \\
\hline Mean tree $\mathrm{DBH}$ & 20.96 & 19.8 & 22.2 & 21.4 & 19.3 & 23.3 \\
\hline Max. tree $\mathrm{DBH}$ & 128.2 & 113.5 & 75.1 & 71.8 & 57.5 & 124.8 \\
\hline Tree species richness & 21 & 30 & 17 & 13 & 24 & 17 \\
\hline Genera & 20 & 29 & 16 & 12 & 22 & 17 \\
\hline Families & 13 & 17 & 11 & 8 & 13 & 12 \\
\hline Soil pH $(0-10 \mathrm{~cm})$ & 6.22 & 6.4 & 6.2 & 5.82 & 6.14 & 6.05 \\
\hline $\begin{array}{l}\text { Soil moisture \% } \\
(0-10 \mathrm{~cm})\end{array}$ & 20.3 & 19.6 & 15.8 & 22.6 & 22.2 & 17.9 \\
\hline Bulk density $\mathrm{g} \mathrm{cm}^{-3}(0-10 \mathrm{~cm})$ & 1.06 & 1.12 & 1.17 & 0.89 & 0.98 & 1.1 \\
\hline
\end{tabular}


A reconnaissance survey of the entire region was carried out, three study sites were selected and each site was divided into two subsites (Lantana-invaded (LI) and uninvaded (UI)). The LI subsites were selected, having Lantana cover/density $>50 \%$. The phytosociological analysis was done in peak growing period during August to October in 2017 and 2018. In each subsite, 10 square plots of $50 \mathrm{~m} \times 50 \mathrm{~m}$ were laid randomly in LI and UI localities. The UI plots were chosen in neighbouring localities at > 50-100 m away from LI plots with similar site conditions. Each $50 \mathrm{~m} \times 50 \mathrm{~m}$ plot was further sub-gridded into 25 (10 m $\times 10 \mathrm{~m}), 10(5 \mathrm{~m} \times 5 \mathrm{~m})$ and $10(1 \mathrm{~m} \times 1 \mathrm{~m})$ quadrats for trees, shrubs and herbs, respectively (Kershaw 1964; Misra 1968). The enumerated individuals of trees were classified into three categories: saplings ( $<3 \mathrm{~cm} \mathrm{DBH}$, diameter at breast height), juveniles $(>3-9.9 \mathrm{~cm} \mathrm{DBH})$, and adults $(\geq 10 \mathrm{~cm} \mathrm{DBH})$. A total of 60 plots $(50 \mathrm{~m} \times 50 \mathrm{~m})$ were laid for trees, $60(5 \mathrm{~m} \times 5 \mathrm{~m})$ for shrubs and herbs respectively (10 in each subsite). In each quardrat, all the tree individuals $>$ $3 \mathrm{~cm} \mathrm{DBH}$ at $1.37 \mathrm{~m}$ above the ground were measured, shrub diameter was recorded at $>10 \mathrm{~cm}$ above the ground level and individuals were counted. Herbaceous species individuals were counted and measured with digital Vernier caliper. Vegetation composition was evaluated by analysing the density (D), basal area (BA), abundance, frequency, and Importance Value Index (IVI) following Misra (1968) and Curtis and Mclntosh (1951). Diameter class-wise distributions were calculated for each subsite. The plant specimens were prepared and identified at Department of Botany, Dr. Harisingh Gour Vishwavidyalaya (A Central University), Sagar, with the help of Flora of Bhopal (Oommachan, 1977) and other standard floras. The assignment of a species to the family was done as per Angiosperm Phylogeny Group IV (APG IV) system of classification (Stevens, 2017). The plant names and the corresponding author citations were given following GRIN (Germplasm Resources Information Network) Taxonomy and Global Biodiversity Information Facility (Wiersema, 2019).

\section{Study sites and soil sampling}

From each plot, soil samples were collected at five random points to a depth of $10 \mathrm{~cm}$ using a soil core sampler of $5 \mathrm{~cm}$ internal diameter. These samples were mixed thoroughly, and $250 \mathrm{~g}$ were collected, air dried, and stored in airtight polyethylene bags and sent to the laboratory for further analysis. The composite soil samples were sieved through a $2 \mathrm{~mm}$ stainless steel sieve using a mortar and pestle. Soil organic carbon (SOC) was estimated following Walkley and Black's method (Walkley 1934), which is a widely used procedure (Pearson et al. 2005). The total soil nitrogen (STN) was calculated using semimicro Kjeldahl method (Kirk 1950).

Soil bulk density was calculated by following Pearson et al. (2005).

$$
\text { Bulkdensity }\left(\mathrm{g} / \mathrm{m}^{3}\right)=\frac{\text { Ovendrymass }\left(\mathrm{g} / \mathrm{m}^{3}\right)}{\text { Corevolume }\left(\mathrm{m}^{3}\right)-\left(\operatorname{Massofcoarsefragments}(\mathrm{g}) / 2.65\left(\mathrm{~g} / \mathrm{cm}^{3}\right)\right.}
$$

where 2.65 was taken as a constant for the density of rock fragments $\left(\mathrm{g} / \mathrm{cm}^{3}\right)$

The total $\mathrm{C}$ content of $0-10 \mathrm{~cm}$ soil depth was estimated by following the formula of Pearson et al. (2005):

$\operatorname{SOC}(\mathrm{Mg} \mathrm{C} / \mathrm{ha})=\left[\left(\right.\right.$ soil bulk density $\left(\mathrm{g} / \mathrm{m}^{3}\right) \times$ soil depth $\left.\left.(\mathrm{cm}) \times \mathrm{C}\right)\right] \times 100$

Soil moisture (\%) was measured by the gravimetric method. Soil pH (1:2.5 ratio of soil: water) was measured with digital pH meter. Three replicates were tested for each forest plot (30 each subsite).

\section{Statistical analysis}

Statistical analysis was done by analysis of variance (one-way ANOVA). Differences in means of species richness, density, basal area, diversity indices and soil properties were tested by Tukey's HSD test (at $p<0.05$ ) using SPSS version 20.0. Diversity indices and box plots were computed and drawn using the Past 3.1 program (version 3.1; Øyvind Hammer, Natural History Museum, University of Oslo). The linear correlation regression was done for assessing the relationship between Lantana densities with soil parameters and other predictor variables.

\section{Results}

\section{Impact on species richness and diversity}

In total, 141 plant species (49 trees, 78 herbs, 14 shrubs and lianas) from 122 genera and 44 families including L. camara were documented in all the three study sites (Table 2). The total species richness (SR) was significantly $(p<0.05)$ lower in LI sites (98) than in UI sites (132). In LI sites (Kesli, Deori and Shahgarh), the SR of sapling, juvenile and adult trees ranged from 1-5, 3-15 and 3-14, whereas in Ul sites it ranged from 1-9, 2-14 and 4-16, respectively. The mean SR of sapling (13), juvenile (30) and adult trees (29) were significantly $(p<0.05)$ lower in LI sites than the sapling (21), juvenile (33) and adult trees (40) in UI sites. The herbaceous SR ranged from 16-36 and 12-26 in UI and LI sites respectively, and the mean SR was significantly ( $<<0.001)$ lower in LI (53) than UI (72) sites, whereas, the SR of shrubs and lianas were also significantly $(p<0.05)$ lower in LI sites (7) than UI sites (13). The total SR in LI sites were 55,72 and 54 , and in $\mathrm{UI}$ sites were 71,98 and 76 for saplings, juveniles and adults respectively (Figs. $3-7$ ). A reduction of $-33,-8,-26,-25,-43$ and $-24 \%$ in SR of saplings, juveniles, adults, herbs, shrubs \& lianas and total SR respectively, has been observed in LI sites compared to UI sites (Fig. 2). Eighty nine species (63.1\%) were found common to both UI and LI sites, while 43 species (30.5\%) occur only in Ul sites and 9 species (6.4\%) only in LI sites (Table 3). 
Table 2

Density $\left(\right.$ No. ha $\left.\mathrm{a}^{-1}\right)$, basal area $\left(\mathrm{m}^{2} \mathrm{ha}^{-1}\right)$ and Importance Value Index (IVI) of different life forms in uninvaded (UI) and Lantana-invaded (LI) sites of tri

\begin{tabular}{|c|c|c|c|c|c|c|c|c|c|c|c|c|}
\hline \multirow{3}{*}{$\begin{array}{l}\text { Life form } \\
\text { Saplings }\end{array}$} & \multicolumn{6}{|c|}{ Density } & \multicolumn{6}{|c|}{ Basal area } \\
\hline & \multicolumn{3}{|c|}{ Uninvaded (UI) } & \multicolumn{3}{|c|}{ Lantana-invaded (ㄴI) } & \multicolumn{3}{|c|}{ Uninvaded (UI) } & \multicolumn{3}{|c|}{ Lantana-invaded (LI) } \\
\hline & Site-1 & Site-2 & Site-3 & Site-1 & Site-2 & Site-3 & Site-1 & Site-2 & Site-3 & Site-1 & Site-2 & Site-3 \\
\hline Acacia catechu (L.f.) Willd. & & & & & 1 & & & & & & 0.0004 & \\
\hline $\begin{array}{l}\text { Acacia leucophloea } \\
\text { (Roxb.)Willd. }\end{array}$ & & 1 & & & 1 & & & 0.0004 & & & 0.0003 & \\
\hline Aegle marmelos (L.) Correa & & 2 & 1 & & & & & 0.0010 & 0.0003 & & & \\
\hline $\begin{array}{l}\text { Anogeissus latifolia (DC.) } \\
\text { Wallich ex Guill. \& Perr. }\end{array}$ & 6 & & 1 & & & & 0.0034 & & 0.0003 & & & \\
\hline Bauhinia racemosa Lam. & & & & & 1 & & & & & & 0.0005 & \\
\hline Bridelia retusa (L.) A.Juss. & & 1 & & & & & & 0.0004 & & & & \\
\hline Buchanania lanzan Spreng & & 3 & & & & & & 0.0014 & & & & \\
\hline $\begin{array}{l}\text { Butea monosperma (Lam.) } \\
\text { Taub. }\end{array}$ & 3 & 1 & & & 2 & & 0.0019 & 0.0003 & & & 0.0006 & \\
\hline Cassia fistula $\mathrm{L}$. & & 5 & 4 & & 6 & 6 & & 0.0022 & 0.0014 & & 0.0026 & 0.003 \\
\hline Dalbergia paniculata (Roxb) & & 6 & & & & & & 0.0022 & & & & \\
\hline Diospyros melanoxylon Roxb & 10 & 75 & 2 & 2 & 24 & 1 & 0.0058 & 0.0358 & 0.0010 & 0.0017 & 0.0116 & 0.000 \\
\hline $\begin{array}{l}\text { Flacourtia indica (Burm. f.) } \\
\text { Merr. }\end{array}$ & & & 2 & & & & & & 0.0010 & & & \\
\hline Garuga pinnata Roxb. & 9 & & & 2 & & & 0.0043 & & & 0.0010 & & \\
\hline $\begin{array}{l}\text { Grewia tiliifolia Vahl. var. } \\
\text { tilifolia }\end{array}$ & & 2 & & & & & & 0.0007 & & & & \\
\hline Kydia calycina Roxb. & & 1 & & & & & & 0.0005 & & & & \\
\hline Lagerstroemia parviflora Roxb & 6 & 6 & 2 & 2 & & 1 & 0.0031 & 0.0030 & 0.0015 & 0.0010 & & 0.000 \\
\hline $\begin{array}{l}\text { Miliusa tomentosa (Roxb.) J. } \\
\text { Sinclair }\end{array}$ & 12 & 45 & & & 3 & 2 & 0.0069 & 0.0222 & & & 0.0018 & 0.001 \\
\hline $\begin{array}{l}\text { Ougeinia oojeinensis } \\
\text { (Roxb.)Hochr. }\end{array}$ & & 2 & & & & & & 0.0011 & & & & \\
\hline $\begin{array}{l}\text { Schleichera oleosa (Lour.) } \\
\text { Oken. }\end{array}$ & & 4 & & & 1 & & & 0.0018 & & & 0.0004 & \\
\hline Semecarpus anacardium L.f. & 1 & & & & & & 0.0004 & & & & & \\
\hline Syzygium cumini (L.) Skeel & & 42 & & & & & & 0.0206 & & & & \\
\hline Tectona grandis L.f. & 26 & 26 & 21 & 1 & 8 & 17 & 0.0131 & 0.0117 & 0.0115 & 0.0006 & 0.0040 & 0.007 \\
\hline $\begin{array}{l}\text { Terminalia tomentosa Wight \& } \\
\text { Arn. }\end{array}$ & & & & & & 2 & & & & & & 0.000 \\
\hline Wrightia tinctora(Roxb) & & & 1 & & 4 & 3 & & & 0.0005 & & 0.0022 & 0.001 \\
\hline \multicolumn{13}{|l|}{ Juveniles } \\
\hline Acacia catechu (L.f.) Willd. & & & 2 & 2 & 3 & & & & 0.0082 & 0.0102 & 0.0034 & \\
\hline $\begin{array}{l}\text { Acacia leucophloea } \\
\text { (Roxb.)Willd. }\end{array}$ & 7 & 3 & 1 & 13 & 3 & & 0.0249 & 0.0079 & 0.0043 & 0.0446 & 0.0110 & \\
\hline Aegle marmelos (L.) Correa & & 1 & 9 & 2 & 4 & 3 & & 0.0017 & 0.0302 & 0.0071 & 0.0081 & 0.020 \\
\hline Annona squamosa $\mathrm{L}$. & & 9 & & & & & & 0.0195 & & & & \\
\hline $\begin{array}{l}\text { Anogeissus latifolia (DC.) } \\
\text { Wallich ex Guill. \& Perr. }\end{array}$ & 24 & 0 & 3 & 23 & 1 & & 0.0716 & & 0.0101 & 0.0890 & 0.0006 & \\
\hline Azadirachta indica A.Juss. & & & & 1 & & & & & & 0.0015 & & \\
\hline Bauhinia racemosa Lam. & 1 & 8 & 1 & 2 & 3 & & 0.0024 & 0.0370 & 0.0031 & 0.0060 & 0.0112 & \\
\hline Bridelia retusa (L.) A.Juss. & 2 & 2 & & 2 & & & 0.0024 & 0.0122 & & 0.0118 & & \\
\hline Buchanania lanzan Spreng & 4 & 17 & 7 & 0 & 7 & 1 & 0.0213 & 0.0665 & 0.0236 & & 0.0361 & 0.004 \\
\hline
\end{tabular}




\begin{tabular}{|c|c|c|c|c|c|c|c|c|c|c|c|c|}
\hline \multirow{3}{*}{$\begin{array}{l}\text { Life form } \\
\begin{array}{l}\text { Butea monosperma (Lam.) } \\
\text { Taub. }\end{array}\end{array}$} & \multicolumn{6}{|c|}{ Density } & \multicolumn{6}{|c|}{ Basal area } \\
\hline & \multicolumn{3}{|c|}{ Uninvaded (UI) } & \multicolumn{3}{|c|}{ Lantana-invaded (LI) } & \multicolumn{3}{|c|}{ Uninvaded (UI) } & \multicolumn{3}{|c|}{ Lantana-invaded (LI) } \\
\hline & \multicolumn{2}{|l|}{94} & \multirow[t]{2}{*}{11} & \multirow[t]{2}{*}{28} & \multirow{2}{*}{$\begin{array}{l}9 \\
1\end{array}$} & 10 & \multicolumn{2}{|l|}{0.2992} & \multirow[t]{2}{*}{0.0366} & \multirow[t]{2}{*}{0.0968} & \multirow{2}{*}{$\begin{array}{l}0.0316 \\
0.0010\end{array}$} & \multirow[t]{2}{*}{0.040} \\
\hline Casearia tomentosa Roxb. & & & & & & & & & & & & \\
\hline Cassia fistula $L$. & 5 & 19 & 6 & 2 & 7 & 24 & 0.0078 & 0.0658 & 0.0233 & 0.0055 & 0.0248 & 0.058 \\
\hline $\begin{array}{l}\text { Chloroxylon swietenia (Roxb.) } \\
\text { DC. }\end{array}$ & & & & & 2 & & & & & & 0.0073 & \\
\hline $\begin{array}{l}\text { Cochlospermum religiosum } \\
\text { (L.) Alston }\end{array}$ & & 1 & & & 1 & & & 0.0048 & & & 0.0041 & \\
\hline Cordia myxa L. & & 2 & & & & & & 0.0046 & & & & \\
\hline Dalbergia paniculata (Roxb) & & 2 & & & & & & 0.0061 & & & & \\
\hline Diospyros melanoxylon Roxb & 98 & 453 & 60 & 40 & 233 & 22 & 0.2103 & 1.3520 & 0.2482 & 0.1034 & 0.7482 & 0.091 \\
\hline $\begin{array}{l}\text { Elaedendron Glaucum (Rottb.) } \\
\text { Pers. }\end{array}$ & & 1 & & & & & & 0.0052 & & & & \\
\hline Ficus glomerata Roxb. & & 3 & & & 2 & & & 0.0139 & & & 0.0022 & \\
\hline $\begin{array}{l}\text { Flacourtia indica (Burm. f.) } \\
\text { Merr. }\end{array}$ & & & 18 & & & 5 & & & 0.0681 & & & 0.019 \\
\hline Garuga pinnata Roxb. & 22 & & & 19 & 3 & & 0.0284 & & & 0.0453 & 0.0097 & \\
\hline $\begin{array}{l}\text { Grewia tiliifolia Vahl. var. } \\
\text { tilifolia }\end{array}$ & & & & & 2 & & & & & & 0.0014 & \\
\hline Holoptelea integrifolia Planch. & 1 & & & 1 & 1 & & 0.0006 & & & 0.0016 & 0.0006 & \\
\hline Kydia calycina Roxb. & & 1 & & & & & & 0.0013 & & & & \\
\hline Lagerstroemia parviflora Roxb. & 42 & 32 & 19 & 74 & 4 & 17 & 0.1055 & 0.0884 & 0.0706 & 0.2519 & 0.0140 & 0.048 \\
\hline $\begin{array}{l}\text { Lannea coromandelica } \\
\text { (Houtt.)Merr. }\end{array}$ & 1 & & & & 1 & & 0.0031 & & & & 0.0059 & \\
\hline Madhuca indica J.F.Gmel. & 1 & & & & 1 & & 0.0024 & & & & 0.0031 & \\
\hline $\begin{array}{l}\text { Miliusa tomentosa (Roxb.) J. } \\
\text { Sinclair }\end{array}$ & 53 & 144 & & 20 & 52 & 6 & 0.0916 & 0.4086 & & 0.0548 & 0.1520 & 0.011 \\
\hline $\begin{array}{l}\text { Ougeinia oojeinensis } \\
\text { (Roxb.)Hochr. }\end{array}$ & & & & & & 2 & & & & & & 0.007 \\
\hline $\begin{array}{l}\text { Schleichera oleosa (Lour.) } \\
\text { Oken. }\end{array}$ & 2 & 18 & & 1 & 2 & & 0.0046 & 0.0531 & & 0.0043 & 0.0055 & \\
\hline Semecarpus anacardium L.f. & 14 & 1 & & 7 & & & 0.0413 & 0.0020 & & 0.0219 & & \\
\hline Syzygium cumini (L.) Skeel & & 84 & & & & & & 0.1953 & & & & \\
\hline Tectona grandis L.f. & 278 & 171 & 75 & 134 & 197 & 102 & 1.0394 & 0.5264 & 0.2020 & 0.5010 & 0.6474 & 0.275 \\
\hline $\begin{array}{l}\text { Terminalia arjuna (Roxb.) } \\
\text { Wight \& Arn. }\end{array}$ & & 2 & & & & & & 0.0074 & & & & \\
\hline $\begin{array}{l}\text { Terminalia tomentosa Wight \& } \\
\text { Arn. }\end{array}$ & 18 & 5 & 2 & 3 & 1 & 2 & 0.0555 & 0.0204 & 0.0056 & 0.0134 & 0.0023 & 0.005 \\
\hline Wrightia tinctora (Roxb.) & & & 10 & & 4 & 6 & & & 0.0267 & & 0.0053 & 0.018 \\
\hline Ziziphus jujuba Mill. & & & 1 & & & & & & 0.0035 & & & \\
\hline $\begin{array}{l}\text { Ziziphus xylopyrus (Retz.) } \\
\text { Willd. }\end{array}$ & & 1 & & 2 & 2 & 1 & & 0.0050 & & 0.0058 & 0.0069 & 0.000 \\
\hline Adults & & & & & & & & & & & & \\
\hline Acacia catechu (L.f.) Willd. & 2 & & 1 & 2 & 2 & & 0.0406 & & 0.0198 & 0.0131 & 0.1006 & \\
\hline $\begin{array}{l}\text { Acacia leucophloea (Roxb.) } \\
\text { Willd. }\end{array}$ & 1 & 6 & 2 & 2 & 10 & 2 & 0.0333 & 0.2878 & 0.2420 & 0.0435 & 0.5120 & 0.098 \\
\hline $\begin{array}{l}\text { Adina cordifolia } \\
\text { (Roxb.) Brandis }\end{array}$ & & 1 & & & & & & 0.0360 & & & & \\
\hline Aegle marmelos (L.) Correa & 2 & 1 & 11 & & & 18 & 0.0404 & 0.0746 & 0.4550 & & & 0.839 \\
\hline
\end{tabular}




\begin{tabular}{|c|c|c|c|c|c|c|c|c|c|c|c|c|}
\hline \multirow{3}{*}{$\begin{array}{l}\text { Life form } \\
\text { Albizia procera (Roxb.) Benth }\end{array}$} & \multicolumn{6}{|c|}{ Density } & \multicolumn{6}{|c|}{ Basal area } \\
\hline & \multicolumn{3}{|c|}{ Uninvaded (UI) } & \multicolumn{3}{|c|}{ Lantana-invaded (LI) } & \multicolumn{3}{|c|}{ Uninvaded (UI) } & \multicolumn{3}{|c|}{ Lantana-invaded (LI) } \\
\hline & 1 & & & & 1 & & 0.0343 & & & & 0.1178 & \\
\hline $\begin{array}{l}\text { Anogeissus latifolia (DC.) } \\
\text { Wallich ex Guill. \& Perr. }\end{array}$ & 17 & 1 & 20 & 26 & 3 & 2 & 0.3968 & 0.1213 & 0.9170 & 1.0131 & 0.2772 & 0.048 \\
\hline Bauhinia racemosa Lam. & 1 & 13 & & 1 & 6 & & 0.0156 & 0.2771 & & 0.0141 & 0.1747 & \\
\hline Bridelia retusa (L.) A.Juss. & & 2 & & & & & & 0.1049 & & & & \\
\hline Buchanania lanzan Spreng & 8 & 44 & 35 & & 34 & 5 & 0.2773 & 1.1584 & 1.3821 & & 1.1994 & 0.221 \\
\hline $\begin{array}{l}\text { Butea monosperma (Lam.) } \\
\text { Taub. }\end{array}$ & 26 & 10 & 74 & 16 & 15 & 50 & 0.7311 & 0.3445 & 4.3461 & 0.2450 & 0.6686 & 3.910 \\
\hline Cassia fistula $\mathrm{L}$. & & 2 & & & 1 & 4 & & 0.0381 & & & 0.0087 & 0.071 \\
\hline $\begin{array}{l}\text { Chloroxylon swietenia (Roxb.) } \\
\text { DC. }\end{array}$ & & 1 & & & & & & 0.0149 & & & & \\
\hline Cordia myxa L. & & & & & 2 & & & & & & 0.1154 & \\
\hline Dalbergia paniculata (Roxb) & 1 & & 4 & & 2 & & 0.2454 & & 0.4004 & & 0.1186 & \\
\hline Diospyros melanoxylon Roxb. & 3 & 78 & 56 & 2 & 53 & 17 & 0.0850 & 1.7044 & 1.7453 & 0.0161 & 1.0829 & 0.433 \\
\hline $\begin{array}{l}\text { Elaedendron Glaucum (Rottb.) } \\
\text { Pers. }\end{array}$ & 1 & & & & & & 0.0097 & & & & & \\
\hline Feronia elephantum Corrêa & & 1 & & & & & & 0.0390 & & & & \\
\hline Ficus glomerata Roxb. & & 5 & & & & & & 0.2366 & & & & \\
\hline Ficus religiosa L. & 1 & & & & 1 & 7 & 1.0329 & & & & 0.0513 & 2.386 \\
\hline $\begin{array}{l}\text { Flacourtia indica (Burm. f.) } \\
\text { Merr. }\end{array}$ & & 1 & 19 & & & 7 & & 0.0336 & 0.3883 & & & 0.228 \\
\hline Gardenia latifolia Aiton & & 1 & & & 5 & & & 0.0602 & & & 0.3101 & \\
\hline Garuga pinnata Roxb. & 2 & & & 1 & 2 & & 0.1690 & & & 0.0103 & 0.0244 & \\
\hline Holoptelea integrifolia Planch. & & 1 & & & & & & 0.0136 & & & & \\
\hline Lagerstroemia parviflora Roxb. & 3 & 16 & 46 & 9 & 1 & 41 & 0.0975 & 0.3672 & 1.8021 & 0.1225 & 0.0454 & 2.032 \\
\hline $\begin{array}{l}\text { Lannea coromandelica } \\
\text { (Houtt.)Merr. }\end{array}$ & 6 & 2 & 2 & 10 & 10 & 6 & 0.4475 & 0.1658 & 0.1873 & 0.6837 & 0.3668 & 0.442 \\
\hline Madhuca indica J.F.Gmel. & & 2 & & & 8 & & & 0.2371 & & & 0.7151 & \\
\hline Mangifera indica L. & & 1 & & & & & & 0.0302 & & & & \\
\hline $\begin{array}{l}\text { Miliusa tomentosa (Roxb.) J. } \\
\text { Sinclair }\end{array}$ & 2 & 38 & & & 15 & 2 & 0.0790 & 0.9851 & & & 0.1783 & 0.033 \\
\hline $\begin{array}{l}\text { Mitragyna parvifolia (Roxb.) } \\
\text { Korth. }\end{array}$ & & 1 & & & & & & 0.0450 & & & & \\
\hline $\begin{array}{l}\text { Ougeinia oojeinensis (Roxb.) } \\
\text { Hochr. }\end{array}$ & & & & & & 3 & & & & & & 0.207 \\
\hline Phyllanthus emblica L. & 2 & & 4 & & & 1 & 0.0166 & & 0.3062 & & & 0.058 \\
\hline Pterocarpus marsupium Roxb. & & & 2 & & 2 & & & & 0.0889 & & 0.1142 & \\
\hline $\begin{array}{l}\text { Schleichera oleosa (Lour.) } \\
\text { Oken. }\end{array}$ & 1 & 11 & & 1 & 6 & & 0.0094 & 0.3928 & & 0.0066 & 0.3262 & \\
\hline Semecarpus anacardium L.f. & 5 & 4 & & 4 & 1 & & 0.1417 & 0.1278 & & 0.1772 & 0.0902 & \\
\hline Syzygium cumini (L.) Skeel & & 10 & & & & & & 0.0137 & & & & \\
\hline Sterculia urens Roxb. & & 1 & & & & & & 0.3702 & & & & \\
\hline Tamarandus indicus $\mathrm{L}$. & & 1 & & & & & & 0.0067 & & & & \\
\hline Tectona grandis L.f. & 438 & 156 & 102 & 268 & 157 & 129 & 18.3109 & 5.3130 & 4.3480 & 12.8335 & 4.6086 & 5.073 \\
\hline $\begin{array}{l}\text { Terminalia arjuna (Roxb.) } \\
\text { Wight \& Arn. }\end{array}$ & & 22 & & & 1 & & & 4.8882 & & & 0.1011 & \\
\hline $\begin{array}{l}\text { Terminalia tomentosa Wight \& } \\
\text { Arn. }\end{array}$ & 5 & 10 & 19 & 1 & 16 & 2 & 0.3219 & 0.7619 & 1.5898 & 0.0078 & 1.0419 & 0.382 \\
\hline
\end{tabular}




\begin{tabular}{|c|c|c|c|c|c|c|c|c|c|c|c|c|}
\hline \multirow{3}{*}{$\begin{array}{l}\text { Life form } \\
\text { Wrightia tinctora (Roxb) }\end{array}$} & \multicolumn{6}{|c|}{ Density } & \multicolumn{6}{|c|}{ Basal area } \\
\hline & \multicolumn{3}{|c|}{ Uninvaded (UI) } & \multicolumn{3}{|c|}{ Lantana-invaded (LI) } & \multicolumn{2}{|c|}{ Uninvaded (UI) } & \multicolumn{4}{|c|}{ Lantana-invaded (LI) } \\
\hline & & & 2 & & & 4 & & & 0.0383 & & & 0.059 \\
\hline Ziziphus xylopyrus (Retz.)Wild. & & & 1 & & & & & & 0.0076 & & & \\
\hline \multicolumn{13}{|l|}{ Shrubs and Lianas } \\
\hline Adhatoda vasica Nees & & 32 & & & & & & 0.0015 & & & & \\
\hline $\begin{array}{l}\text { Carissa } \\
\text { spinarum var. spinarum }\end{array}$ & & & 64 & & & & & & 0.0048 & & & \\
\hline Cayratia triflora (L.) Domin & & & 48 & & & 256 & & & 0.0004 & & & 0.001 \\
\hline $\begin{array}{l}\text { Desmodium gangeticum } \\
\text { (L.)DC. }\end{array}$ & & 576 & 32 & & & & & 0.0027 & 0.0002 & & & \\
\hline Helicteres isora $\mathrm{L}$. & & 32 & & & & & & 0.0064 & & & & \\
\hline Hemidesmus indicus (L.) R. Br. & 576 & 2480 & 1328 & 496 & 624 & 704 & 0.0146 & 0.0556 & 0.0362 & 0.0075 & 0.0138 & 0.018 \\
\hline $\begin{array}{l}\text { Ichnocarpus frutescens (L.) W. } \\
\text { T. Aiton (C) }\end{array}$ & & & & & 416 & 496 & & & & & 0.0101 & 0.016 \\
\hline Lantana camara L. & 96 & 112 & 128 & 16176 & 17440 & 14512 & 0.0090 & 0.0138 & 0.0437 & 2.6001 & 2.5138 & 1.622 \\
\hline Maytenus emarginata (Willd.) & 32 & 400 & & 32 & 224 & 32 & 0.0068 & 0.5265 & & 0.0033 & 0.2272 & 0.010 \\
\hline Phyllodium pulchellum (L.) & & 144 & & & & & & 0.0077 & & & & \\
\hline Solanum anguivi Lam. & & & 864 & & & & & & 0.0772 & & & \\
\hline Ventilago calyculata Tul. & 16 & 16 & 32 & & 16 & & 0.0771 & 0.0974 & 0.0033 & & 0.0125 & \\
\hline Xanthium strumarium L. & 48 & & 48 & 176 & & & 0.0086 & & 0.0081 & 0.0340 & & \\
\hline Ziziphus oenoplia (L.) Miller & & 16 & & & & & & 0.0199 & & & & \\
\hline \multicolumn{13}{|l|}{ Herbs } \\
\hline $\begin{array}{l}\text { Acanthospermum hispidum } \\
\text { DC. }\end{array}$ & & & & 400 & & & & & & 0.0040 & & \\
\hline Achyranthes aspera L. & 200 & 1000 & 2800 & & 200 & 2400 & 0.0088 & 0.0728 & 0.1944 & & 0.0160 & 0.146 \\
\hline Ageratum conyzoides $\mathrm{L}$. & 30800 & 10800 & 19600 & 7800 & 10000 & 4600 & 0.3592 & 0.2480 & 0.3440 & 0.1296 & 0.2432 & 0.084 \\
\hline $\begin{array}{l}\text { Alternanthera sessilis (L.) R. } \\
\text { Br. ex DC. }\end{array}$ & 2600 & 12400 & & 1600 & 11800 & & 0.0960 & 0.3280 & & 0.0176 & 0.3376 & \\
\hline $\begin{array}{l}\text { Alysicarpus longifolius Sensu } \\
\text { Span., non Wight \& Arn. }\end{array}$ & 4800 & 18400 & & 400 & 3800 & & 0.0360 & 0.1664 & & 0.0024 & 0.0416 & \\
\hline Alysicarpus monilifer (L.) DC. & 5800 & 4200 & & 2400 & 1200 & 1000 & 0.0464 & 0.0360 & & 0.0360 & 0.0112 & 0.007 \\
\hline $\begin{array}{l}\text { Andrographis echioides } \\
\text { (L.) Nees }\end{array}$ & & 3800 & & & 200 & & & 0.0216 & & & 0.0016 & \\
\hline $\begin{array}{l}\text { Anisochilus carnosus } \\
\text { (L.f.) Wall. }\end{array}$ & 800 & & 400 & & & & 0.0160 & & 0.0024 & & & \\
\hline Apluda mutica L. & & 11400 & & 14600 & 1000 & & & 0.0424 & & 0.0488 & 0.0056 & \\
\hline Aristida depressa Retz. & & 11600 & & & & & & 0.0256 & & & & \\
\hline Aristolochia indica L. & & & 200 & & & & & & 0.0104 & & & \\
\hline $\begin{array}{l}\text { Axonopus compressus (Sw.) } \\
\text { P.Beauv. }\end{array}$ & 1000 & & & & & & 0.0088 & & & & & \\
\hline $\begin{array}{l}\text { Bidens biternata } \\
\text { (Lour.) Merr. \& Sherff }\end{array}$ & 6200 & 800 & & 7800 & & & 0.3448 & 0.0432 & & 0.4984 & & \\
\hline $\begin{array}{l}\text { Biophytum sensitivum (L.) DC. } \\
\text { var. sesbanioides }\end{array}$ & 5600 & 10000 & & 1600 & & & 0.1576 & 0.3712 & & 0.0728 & & \\
\hline Blumea lacera (Burm.f.) DC. & & 10200 & & 400 & & & & 0.3320 & & 0.0160 & & \\
\hline $\begin{array}{l}\text { Cajanus scarabaeoides (L.) } \\
\text { Thouars }\end{array}$ & 3400 & & & & & & 0.0360 & & & & & \\
\hline Cassia pumila Lam. & 18000 & 8800 & 600 & 3200 & 600 & 1200 & 0.4592 & 0.1008 & 0.0112 & 0.0336 & 0.0128 & 0.010 \\
\hline Cassia tora L. & 30000 & 4000 & 36800 & 18800 & & 32800 & 1.6424 & 0.1752 & 2.2440 & 1.0200 & & 1.531 \\
\hline
\end{tabular}




\begin{tabular}{|c|c|c|c|c|c|c|c|c|c|c|c|c|}
\hline \multirow{3}{*}{$\begin{array}{l}\text { Life form } \\
\text { Centella asiatica (L.) Urb. }\end{array}$} & \multicolumn{6}{|l|}{ Density } & \multicolumn{6}{|c|}{ Basal area } \\
\hline & \multicolumn{3}{|c|}{ Uninvaded (UI) } & \multicolumn{3}{|c|}{ Lantana-invaded (ㄴI) } & \multicolumn{3}{|c|}{ Uninvaded (UI) } & \multicolumn{3}{|c|}{ Lantana-invaded (LI) } \\
\hline & 600 & & & & & & 0.0008 & & & & & \\
\hline Cissampelos pareira L & 1000 & 5200 & 400 & & 2000 & 1200 & 0.0056 & 0.0472 & 0.0048 & & 0.0272 & 0.011 \\
\hline Cocculus hirsutus (L.) Diels & & 1200 & & & & & & 0.0112 & & & & \\
\hline Commelina benghalensis L. & 200 & 1200 & 600 & 200 & & & 0.0008 & 0.0104 & 0.0064 & 0.0024 & & \\
\hline $\begin{array}{l}\text { Conyza canadensis } \\
\text { (L.) Cronquist }\end{array}$ & & & 200 & & & & & 0.0000 & 0.0008 & & & \\
\hline Corchorus trilocularis L. & 5000 & 4800 & 1200 & & 1000 & 1000 & 0.1616 & 0.1384 & 0.0416 & & 0.0328 & 0.022 \\
\hline Crotolaria juncea L. & & & 200 & & & & & & 0.0128 & & & \\
\hline Curculigo orchioides Gaertn & & 6200 & 1200 & & & 1000 & & 0.1760 & 0.0344 & & & 0.028 \\
\hline Cynodon dactylon (L.) Pers. & 32200 & 20600 & & 5000 & 8000 & & 0.1296 & 0.0936 & & 0.0320 & 0.0432 & \\
\hline Cynoglossum furcatum Wall. & 400 & & & & & & 0.0096 & & & & & \\
\hline Cyperus rotundus $\mathrm{L}$. & 2000 & 1800 & 1200 & 2000 & & 200 & 0.0168 & 0.0136 & 0.0096 & 0.0168 & & 0.003 \\
\hline $\begin{array}{l}\text { Dactyloctenium aegyptium } \\
\text { (L.) Willd. }\end{array}$ & & & 2200 & & & 600 & & & 0.0152 & & & 0.003 \\
\hline Desmodium triflorum (L.) DC. & 72000 & 93000 & 15200 & 34600 & 37000 & 4200 & 0.2912 & 0.4056 & 0.0664 & 0.1384 & 0.1624 & 0.022 \\
\hline $\begin{array}{l}\text { Dichanthium annulatum } \\
\text { (Forssk.) Stapf. }\end{array}$ & 1400 & 1000 & 800 & 2400 & & 600 & 0.0048 & 0.0072 & 0.0040 & 0.0136 & & 0.003 \\
\hline Digitaria sanguinalis (L.) Scop. & & 600 & 2000 & & & & & 0.0024 & 0.0248 & & & \\
\hline $\begin{array}{l}\text { Elephantopus scaber Auct. non } \\
\text { L. }\end{array}$ & 400 & 3200 & 5800 & & 4600 & 4400 & 0.0152 & 0.1400 & 0.2816 & & 0.2000 & 0.194 \\
\hline Eleusine indica (L.) Gaertn. & 5600 & 1000 & 1400 & 1600 & & & 0.0216 & 0.0048 & 0.0112 & 0.0072 & & \\
\hline $\begin{array}{l}\text { Emilia sonchifolia (L.) DC. ex } \\
\text { Wight }\end{array}$ & & & 2200 & & 200 & & & & 0.0080 & & 0.0008 & \\
\hline $\begin{array}{l}\text { Eragrostis unioloides } \\
\text { (Retz.) Nees ex Steud }\end{array}$ & & 2000 & 2200 & & & 1000 & & 0.0064 & 0.0040 & & & 0.001 \\
\hline Euphorbia hirta L. & 10800 & 3600 & 2800 & 9000 & 1000 & 1200 & 0.2208 & 0.0768 & 0.0552 & 0.1656 & 0.0168 & 0.009 \\
\hline Euphorbia hypericifolia L. & & & 1400 & & & & & & 0.0160 & & & \\
\hline Evolvulus alsinoides (L.) & & & 400 & & & & & & 0.0024 & & & \\
\hline Evolvulus nummularius (L.) & & & 71200 & & 1600 & 35200 & & & 0.1712 & & 0.0088 & 0.092 \\
\hline $\begin{array}{l}\text { Heteropogon contortus } \\
\text { (L.) P.Beauv. ex Roem. \& Schult. }\end{array}$ & 12000 & 19200 & & 7400 & 15800 & & 0.1112 & 0.1480 & & 0.0448 & 0.1208 & \\
\hline $\begin{array}{l}\text { Hibiscus lobatus } \\
\text { (Murray) Kuntze }\end{array}$ & 1800 & & 400 & & 200 & 200 & 0.0456 & & 0.0160 & & 0.0016 & 0.002 \\
\hline Hyptis suaveolens (L.) Poit. & & 600 & 1600 & 6000 & & & & 0.0288 & 0.0776 & 0.2680 & & \\
\hline Indigofera cordifolia Roth & & 600 & & & & & & 0.0088 & & & & \\
\hline $\begin{array}{l}\text { lonidium suffruticosum } \\
\text { (L.) Ging. in DC. }\end{array}$ & & & & & 400 & & & & & & 0.0048 & \\
\hline Ipomoea cairica (L.) Sweet & & & & & 600 & 600 & & & & & 0.0040 & 0.005 \\
\hline $\begin{array}{l}\text { Justicia quinqueangularis } \\
\text { Koen. ex Roxb. }\end{array}$ & 16000 & 7400 & & 4800 & 1000 & & 0.1368 & 0.0624 & & 0.0480 & 0.0096 & \\
\hline $\begin{array}{l}\text { Lindernia ciliata (Colsm.) } \\
\text { Pennell }\end{array}$ & & & 2200 & & & & & & 0.1152 & & & \\
\hline $\begin{array}{l}\text { Lindernia crustacea (L.) F. } \\
\text { Muell. }\end{array}$ & & & 8600 & & & & & & 0.0568 & & & \\
\hline $\begin{array}{l}\text { Melothria heterophylla (Lour.) } \\
\text { Cogn. }\end{array}$ & 400 & & & & & & 0.0024 & & & & & \\
\hline $\begin{array}{l}\text { Merremia emarginata } \\
\text { (Burm. fil.) Hall. fil. }\end{array}$ & 400 & & & & & & 0.0008 & & & & & \\
\hline Mollugo oppositifolia L. & & & 400 & & 200 & & & & 0.0056 & & 0.0024 & \\
\hline
\end{tabular}




\begin{tabular}{|c|c|c|c|c|c|c|c|c|c|c|c|c|}
\hline \multirow{3}{*}{$\begin{array}{l}\text { Life form } \\
\text { Oldenlandia herbacea (L.) }\end{array}$} & \multicolumn{6}{|l|}{ Density } & \multicolumn{6}{|c|}{ Basal area } \\
\hline & \multicolumn{3}{|c|}{ Uninvaded (UI) } & \multicolumn{3}{|c|}{ Lantana-invaded (LI) } & \multicolumn{3}{|c|}{ Uninvaded (UI) } & \multicolumn{3}{|c|}{ Lantana-invaded (LI) } \\
\hline & & 1200 & & & & & & 0.0024 & & & & \\
\hline $\begin{array}{l}\text { Oplismenus burmannii } \\
\text { (Retz.) P.Beauv. }\end{array}$ & 79600 & 85400 & 120400 & 58800 & 129600 & 95400 & 0.2152 & 0.2048 & 0.3048 & 0.1296 & 0.3296 & 0.220 \\
\hline Parthenium hysterophorus L. & & & 10200 & & & 5000 & & & 0.6856 & & & 0.512 \\
\hline $\begin{array}{l}\text { Peristrophe paniculata } \\
\text { (Forssk.) R.K. Brummitt }\end{array}$ & & 1000 & & & & & & 0.0088 & & & & \\
\hline $\begin{array}{l}\text { Phyllanthus } \\
\text { fraternus G.L.Webster }\end{array}$ & & 800 & 200 & & & & & 0.0136 & 0.0040 & & & \\
\hline Phyllanthus niruri $\mathrm{L}$. & & 200 & 1600 & & & & & 0.0160 & 0.0808 & & & \\
\hline Phyllanthus urinaria L. & 800 & 1200 & 3000 & & & 1000 & 0.0088 & 0.0216 & 0.0192 & & & 0.005 \\
\hline Phyllanthus simplex Retz. & 1200 & 1200 & 6000 & & 200 & 1200 & 0.0144 & 0.0152 & 0.0824 & & 0.0024 & 0.016 \\
\hline Physalis minima L. & & 400 & 400 & & & & & 0.0256 & 0.0104 & & & \\
\hline Polygala chinensis L. & & 1000 & & & & & & 0.0048 & & & & \\
\hline Ruellia tuberosa L. & 1600 & 11400 & 8400 & & 19000 & 6000 & 0.0360 & 0.2032 & 0.1960 & & 0.3408 & 0.160 \\
\hline Rungia pectinata (L.) & 17800 & 46800 & 23000 & 9600 & 30200 & 15600 & 0.1432 & 0.4624 & 0.2536 & 0.1008 & 0.3240 & 0.157 \\
\hline Setaria viridis (L.) P.Beauv. & 400 & 2000 & 1200 & 1200 & & & 0.0024 & 0.0112 & 0.0088 & 0.0048 & & \\
\hline Sida acuta Burm. f. & & & & 7800 & 6400 & 8600 & & & & 0.4400 & 0.3152 & 0.446 \\
\hline Sida cordifolia L. & 26400 & 23600 & 29600 & 20200 & 21000 & 31400 & 1.0152 & 0.5696 & 1.1200 & 0.8640 & 0.5576 & 1.154 \\
\hline Sida rhombifolia L. & & & & & & 2600 & & & & & & 0.119 \\
\hline Spermacoce hispida Linn & & 200 & 2400 & & & & & 0.0024 & 0.0152 & & & \\
\hline Spigelia anthelmia L. & & & 1400 & & & 200 & & & 0.0112 & & & 0.001 \\
\hline $\begin{array}{l}\text { Sporobolus diander } \\
\text { (Retz.) Beauv. }\end{array}$ & 1600 & 3200 & 13000 & 2600 & 3400 & 2000 & 0.0024 & 0.0056 & 0.0184 & 0.0040 & 0.0064 & 0.003 \\
\hline Themeda triandra Forssk. & 12600 & 11200 & & 3400 & 3200 & & 0.0640 & 0.0504 & & 0.0208 & 0.0112 & \\
\hline Tridax procumbens $\mathrm{L}$. & 15000 & 5200 & 800 & 13400 & 3800 & 800 & 0.4792 & 0.2000 & 0.0184 & 0.3712 & 0.0952 & 0.028 \\
\hline Triumfetta rhomboidea Jacq. & 26000 & 9000 & 400 & 17000 & 800 & 1400 & 1.3856 & 0.4912 & 0.0160 & 0.8824 & 0.0288 & 0.048 \\
\hline Urena lobata L. & & & & 1800 & & & & & & 0.4576 & & \\
\hline Vernonia cinera (L.) & 1200 & & 2400 & & 600 & & 0.0304 & & 0.0472 & & 0.0104 & \\
\hline Zornia diphylla (L.)Pers. & & 3400 & 400 & & 400 & & & 0.0160 & 0.0016 & & 0.0032 & \\
\hline
\end{tabular}


Table 3

Family-wise contribution of genera (G), species (S) and density (D; No ha-1) in uninvaded (UI) and Lantana-inavded (LI) sites in tropical dry deciduous forests of Sagar, Madhya Pradesh, India

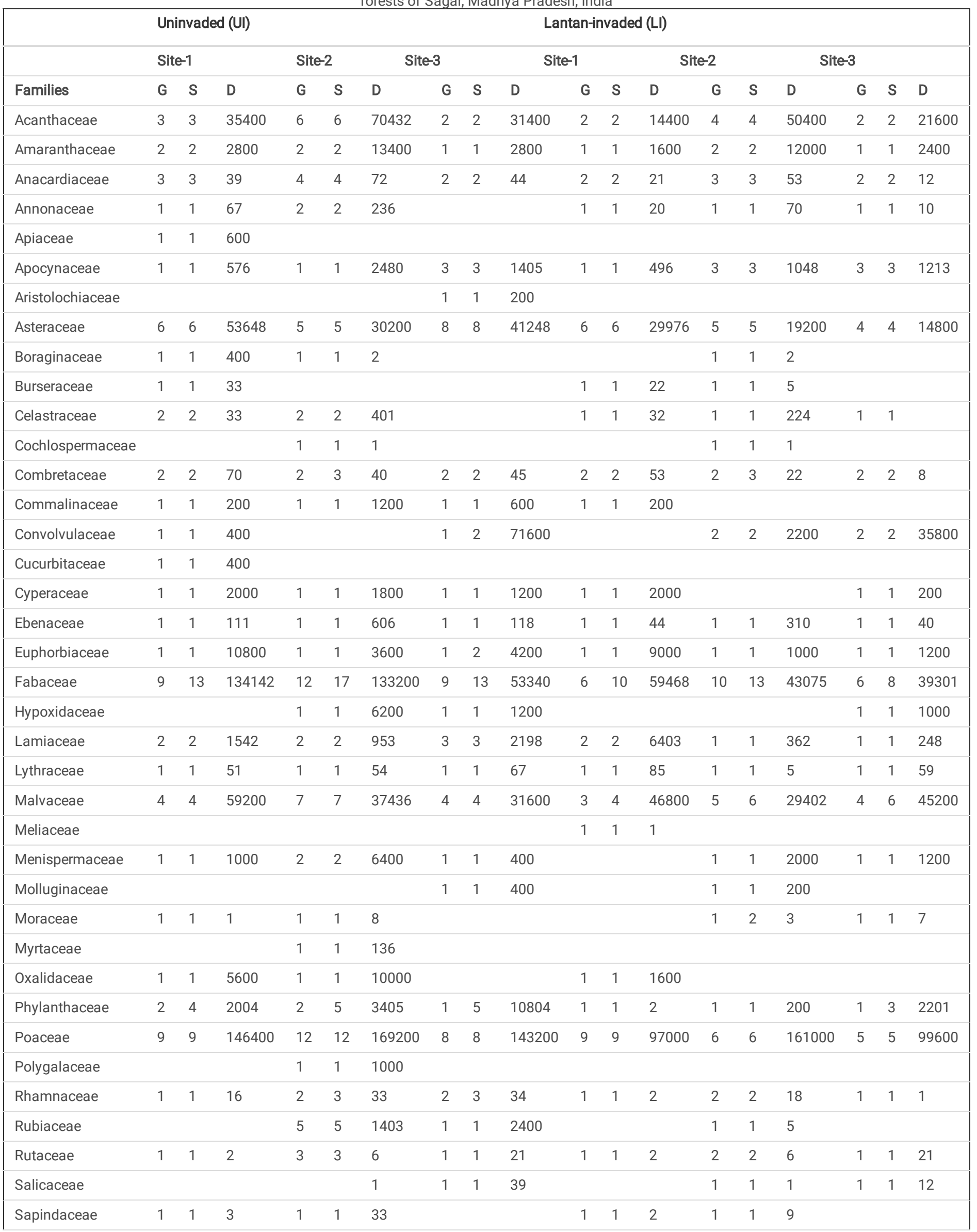




\begin{tabular}{|c|c|c|c|c|c|c|c|c|c|c|c|c|c|c|c|c|c|c|}
\hline \multirow[b]{2}{*}{ Sapotaceae } & \multicolumn{6}{|c|}{ Uninvaded (UI) } & \multicolumn{9}{|c|}{ Lantan-invaded (LI) } & & & \\
\hline & 1 & 1 & 1 & 1 & 1 & 2 & & & & & & & 1 & 1 & 9 & & & \\
\hline Scrophulariaceae & & & & & & & 1 & 2 & 10800 & & & & & & & & & \\
\hline Solanaceae & & & & 1 & 1 & 400 & 2 & 2 & 1264 & & & & & & & & & \\
\hline Spigeliaceae & & & & & & & 1 & 1 & 1400 & & & & & & & 1 & 1 & 200 \\
\hline Ulmaceae & 1 & 1 & 1 & 1 & 1 & 1 & & & & 1 & 1 & 1 & 1 & 1 & 1 & & & \\
\hline Verbenaceae & 1 & 1 & 96 & 1 & 1 & 112 & 1 & 1 & 128 & 1 & 1 & 16176 & 1 & 1 & 17440 & 1 & 1 & 14512 \\
\hline Violaceae & & & & & & & & & & & & & 1 & 1 & 400 & & & \\
\hline Vitaceae & & & & & & & 1 & 1 & 48 & & & & & & & 1 & 1 & 256 \\
\hline
\end{tabular}

The Shannon's index $(\mathrm{H} \mathrm{X})$ ranged from $0.35-1.81,0.41-2.00$ and $0.46-2.12$ in Ul sites and from $0.51-1.61,0.66-2.08$ and $0.50-1.91$ in $\mathrm{LI}$ sites, for saplings, juveniles and adults, respectively, and the values were significantly $(\mathrm{p}<0.05)$ lower in LI sites than in Ul sites (Figs. $3-7)$. For herbs the values ranged from $1.77-3.03$ and $1.80-2.60$ in UI and LI sites respectively. Significantly $(p<0.05)$ lower Shannon's index has been observed in LI than in UI sites. Simpson's dominance index (Cd) of saplings, juveniles and adult tree species ranged from $0.19-0.80,0.18-0.76$ and $0.14-0.83$ in $\mathrm{Ul}$ sites and $0.20-0.65,0.16-0.64$ and $0.21-0.76$ in LI sites respectively, and the values were significantly $(p<0.05)$ lower in LI than UI sites (Figs. $3-7)$. In case of herbs, it ranged from $0.06-0.25$ and $0.09-0.30 \mathrm{in} \mathrm{Ul}$ and $\mathrm{LI}$ sites respectively. Whereas, the evenness index (E) of saplings, juveniles and adult tree species ranged from $0.45-0.89,0.28-0.75$ and $0.18-0.69$ in Ul sites and $0.60-0.95,0.29-0.77$ and $0.31-0.79$ in LI sites respectively. The highest evenness has been observed in LI than in UI sites. Herb evenness ranged from $0.33-0.71$ and $0.33-0.76$ in $\mathrm{UI}$ and LI sites, respectively and was significantly higher in LI sites than in UI sites (Figs. 3-7). The Margalef's index $(R)$ of tree saplings, herbs and total plants reduced significantly $(p<0.00)$ in LI than Ul sites (Figs. $3-7)$.

\section{Impact on density and basal area}

A significant impact of Lantana density (No. ha ${ }^{-1}$ ) has been observed on the density (No. ha $\left.{ }^{-1}\right)$ and basal area $\left(\mathrm{m}^{2} \mathrm{ha}^{-1}\right)$ of trees and herbaceous vegetation in LI than UI sites (Table 2). The density of tree saplings and juveniles decreased significantly $(\mathrm{p}<0.001)$ with increase in Lantana density, whereas, the adult tree density did not show any significant trend (Figs. 2-6). The tree density of saplings and juveniles ranged from 4-308 and 4-100 in UI and 104-1336 and $132-732$ in LI sites, respectively. The mean density of saplings and juveniles reduced significantly ( $p<0.001)$ and was highest in UI (109 \& 623$)$ than LI (29 \& 373) sites. The adult tree density ranged from 312-664 and 248-424 in UI and LI sites, with the mean of 456 and 331 respectively. The total tree density (saplings + juveniles and adults) also showed a significant $(\mathrm{p}<0.001)$ declining trend in LI than UI sites. The total tree density in LI sites reduced significantly $(p<0.05)$ and ranged from 452-1116 and in Ul sites from 528-1960, with the mean of 734 and 1118, respectively. The mean herb density declined significantly $(p<0.001)$ in LI sites than UI sites. The herb density ranged from $289000-640000$ and $188000-373000$ in UI and LI sites, with the mean of 451866 and 284466 , respectively. ). In total a reduction of $-53,-25,-16,-23$, and $-20 \%$ in density of saplings, juveniles, adults, herbs and total $D$ respectively, has been observed in LI sites compared to UI sites, except shrubs \& lianas density, which was having 76\% higher density in LI than UI sites (Fig. 2).

The basal area of saplings and juveniles decreased significantly $(\mathrm{p}<0.001)$ with increase in Lantana density, whereas, the adult basal area did not show any significant trend. The basal area of saplings and juveniles declined significantly $(p<0.001)$ in LI sites and ranged from $0.00-0.02$ and $0.34-2.09$ in LI sites, and $0.01-0.15$ and $0.43-4.29$ in Ul sites, with the mean basal area of 0.05 and 1.89, and 0.01 and 1.21 respectively. The adult tree basal ranged from 14.530.3 and 11.5-23.4 in UI and LI sites with the mean of 19.7 and 14.7, respectively (Figs. 2-6). The total tree basal area reduced significantly ( $<<0.01$ ) in LI sites and ranged from 13.5-24.1 and in UI sites from 15.2-33.1, with the mean of 15.9 and 21.6, respectively. The mean herb basal area declined significantly $(\mathrm{p}<0.001)$ in LI sites than in UI sites. The herb basal area ranged from 4.3-9.2 and 2.6-7.1 in UI and LI sites, with the mean of 6.7 and 4.8 respectively. In total a reduction of $-67,-22,-15,-18$, and $-11 \%$ in basal area of saplings, juveniles, adults, herbs and total $\mathrm{D}$ respectively, has been observed in $\mathrm{LI}$ sites than $\mathrm{UI}$ sites, except shrubs \& lianas, which are having $78 \%$ higher basal area in LI than UI sites (Fig. 2).

\section{Impact on family composition}

The number of species in a family varied from 1-17 and 1-13 in UI and LI sites respectively. Out of 141 species, 88 species from 35 families were found to be common in both $\mathrm{UI}$ and $\mathrm{LI}$ sites. Forty four species from 7 families and 10 species from 2 families were found only in $\mathrm{UI}$ and LI sites respectively. Fabaceae (17(UI) and 13(LI) species), Poaceae (12(UI) and 9(LI), Asteraceae (8(UI) and 6(LI) species), Malvaceae (7(UI) and 6(LI) species) and Acanthaceae (6(UI) and 4(LI) species) were the most speciose families (Table 3).

\section{Impact on diameter class distribution}

Tree density decreased significantly $(\mathrm{p}<0.001)$ with increase in diameter class in both UI and LI sites. The highest density of $51.8 \%$ was contributed by $3.1-$ $10 \mathrm{~cm}$ size class and lowest of $0.7 \%$ density was contributed by $>50 \mathrm{~cm}$ size class in both UI and LI sites. Tree density of lower diameter class (<3 DBH) was reduced by $57 \%$ in LI sites followed by $25,23,16,13$ and $10 \%$ of $3.1-10,30.1-40,10.1-20,20.1-30$ and $40.1-50$ DBH classes respectively (Fig. 8). In both LI and $\mathrm{UI}$ sites, the first three DBH classes $(<3-20 \mathrm{~cm})$ contributed $81.8 \%$ of the total density, whereas, the other four DBH classes ( $>20 \mathrm{~cm}) \mathrm{contributed} 18.2 \%$ of the total density.

Impact on the soil properties 
The values of soil bulk density $\left(\mathrm{g} \mathrm{cm}^{-3}\right)$ and $\mathrm{pH}$ were significantly $(F=12.96, \mathrm{p}<0.05)$ lower in LI than in UI sites, whereas, soil moisture $(\%)$ was significantly $(F=68.55, \mathrm{p}<0.001)$ higher in LI than in UI sites (Fig. 9). The SOC and STN stocks were significantly $(F=48.3, p<0.001 ; F=51.7, \mathrm{p}<0.001)$ higher in LI than in Ul sites (Fig. 9).

\section{Correlation between Lantana densities with other different variables}

The species richness of tree seedlings, juveniles and herbs showed a significant negative correlation $(r=-0.261$ to $-0.720, p<0.001$, Table 4$)$ with $L a n t a n a$ density, whereas, a non-significant negative correlation was observed in SR of adult and total trees with Lantana densities. The density and basal area of tree seedlings, juveniles and herbs were significantly negatively correlated with Lantana density. Similarly, diversity indices (Simpson index, Shannon index,

Fisher's alpha and Margalef's index) also showed a significant negative correlation with Lantana density, whereas, the evenness index of adult trees and herbs were significantly negatively correlated with Lantana density. The SOC, STN and M (\%) showed a significant positive correlation, whereas, soil bulk density and $\mathrm{pH}$ showed a significant negative correlation with Lantana densities (Table 4).

Table 4

Correlations ( $r$ - values) between Lantana density and other predictable variables

\begin{tabular}{|c|c|c|c|c|c|}
\hline Predictor variables & Fvalue & $P$-value & Predictor variables & f-value & $P$-value \\
\hline \multicolumn{3}{|l|}{ Species richness } & \multicolumn{3}{|c|}{ Density (No. of individuals ha-1) } \\
\hline Tree seedlings & -0.720 & 0.001 & Tree seedlings & -0.867 & 0.001 \\
\hline Tree juveniles & -0.280 & 0.051 & Tree juveniles & -0.312 & 0.023 \\
\hline Tree adults & -0.261 & 0.347 & Tree adults & 0.369 & 0.176 \\
\hline Total trees & -0.110 & 0.696 & Total trees & -0.288 & 0.298 \\
\hline Herb & -0.385 & 0.044 & Herb & -0.685 & 0.013 \\
\hline \multicolumn{3}{|l|}{ Basal area $\left(\mathrm{m}^{2} \mathrm{ha} \mathrm{a}^{-1}\right)$} & \multicolumn{3}{|l|}{ Shannon index $(H 囚)$} \\
\hline Tree seedlings & -0.887 & 0.001 & Tree seedlings & -0.671 & 0.001 \\
\hline Tree juveniles & -0.168 & 0.045 & Tree juveniles & -0.253 & 0.021 \\
\hline Tree adults & -0.047 & 0.869 & Tree adults & -0.433 & 0.107 \\
\hline Total trees & -0.085 & 0.762 & Total trees & -0.118 & 0.674 \\
\hline Herb & 0.538 & 0.039 & Herb & -0.037 & 0.053 \\
\hline \multicolumn{3}{|l|}{ Dominance index (D) } & \multicolumn{3}{|l|}{ Simpson index (1-D) } \\
\hline Tree seedlings & 0.417 & 0.122 & Tree seedlings & -0.663 & 0.001 \\
\hline Tree juveniles & 0.153 & 0.041 & Tree juveniles & -0.153 & 0.041 \\
\hline Tree adults & 0.493 & 0.062 & Tree adults & -0.493 & 0.062 \\
\hline Total trees & 0.223 & 0.425 & Total trees & -0.223 & 0.425 \\
\hline Herb & 0.044 & 0.035 & Herb & -0.044 & 0.035 \\
\hline \multicolumn{3}{|l|}{ Evenness index (E) } & \multicolumn{3}{|l|}{ Fisher's alpha (a) } \\
\hline Tree seedlings & 0.033 & 0.908 & Tree seedlings & -0.445 & 0.096 \\
\hline Tree juveniles & -0.054 & 0.848 & Tree juveniles & -0.389 & 0.032 \\
\hline Tree adults & -0.472 & 0.076 & Tree adults & -0.311 & 0.259 \\
\hline Total trees & -0.098 & 0.729 & Total trees & -0.070 & 0.803 \\
\hline Herb & 0.606 & 0.017 & Herb & -0.028 & 0.024 \\
\hline \multicolumn{3}{|l|}{ Margalefs index (R) } & \multicolumn{3}{|l|}{ Soil properties } \\
\hline Tree seedlings & -0.613 & 0.015 & soc & 0.890 & 0.043 \\
\hline Tree juveniles & -0.368 & 0.05 & $\mathrm{~N}$ & 0.830 & 0.052 \\
\hline Tree adults & -0.291 & 0.292 & $\mathrm{pH}$ & -0.987 & 0.002 \\
\hline Total trees & -0.072 & 0.799 & M (\%) & 0.432 & 0.001 \\
\hline Herb & -0.024 & 0.035 & $\mathrm{BD}$ & -0.513 & 0.051 \\
\hline
\end{tabular}

\section{Discussion}


Comparing invaded and uninvaded sites aid us to measure the impact of invasive species on the native resident communities (Levine et al. 2003). In the present study, the LI sites had a significantly $(\mathrm{p}<0.05)$ lower SR than the UI sites. Localities with Lantana invasion are known to have lower SR than uninvaded localities (Sharma and Raghubanshi 2010; Dobhal et al. 2011; Kumar et al. 2020). It is therefore evident that Lantana successfully establishes itself and competitively excludes the native resident plant communities in the studied sites. The variation in SR among the sites could be because native species differ in their resistance to invasion where some species are more easily excluded than the others in invaded sites (Stinson et al. 2007). About $33,8,26,25,43$ and $24 \%$ decline in SR were observed in saplings, juveniles, adults, herbs, shrubs \& lianas and total species respectively in LI sites compared to UI sites. Increase in Lantana cover is known to cause pervasive losses in SR across multiple life forms (Gooden et al. 2009; Sharma and Raghubanshi 2010). The highest decline in SR was observed in the case of tree saplings, juveniles and herbs. This could be because Lantana produces a large number of light-weight seeds with high adaptability that enables them to grow vigorously and suppress the growth of native plant species. Furthermore, the release of allelochemicals from its roots also hampers the growth of native plant community (Kumar et al. 2020).

The values of Shannon index of diversity were lower in LI sites than UI sites for all the life forms and significantly in case of seedlings, juveniles and herbs. Shannon index is a measure of species richness of an ecosystem. Lantana invasion has often been associated with significant decreases in plant species richness, diversity and evenness in deciduous forest types (Sundaram and Hiremath 2012, Badalamenti et al. 2016). In the present study, the evenness index increased in LI sites for tree saplings, but decreased for the shrub and liana category. Changes in evenness of an ecosystem may impact the productivity, resistance to invasion and local plant extinction rates (Wilsey and Potvin 2000; Hejda et al. 2009). The impact of invasion largely depends on the degree of dominance (Pyšek and Pyšek 1995). Overall, the values of dominance index were higher in LI sites than Ul sites. This is concurrent with the view that native plant community suppression often stems from the invasive species dominance in invaded habitats (Richardson et al. 1989). The increase in dominance is often linked to changes in species evenness and richness in invaded habitats (Hejda et al. 2009).

Fabaceae, Poaceae and Asteraceae were the most speciose families in the studied sites. Large families such as these are often the most well-represented and widely distributed in nature (Subashree et al. 2020). A family's dominance largely depends on its species' adaptability and the presence of favourable environmental conditions for pollination, seed dispersal and successful establishment (Panda et al. 2013). However, the number of families was slightly lower in LI sites (39) than UI sites (44). With unabated invasion of Lantana in the studied sites, the number of families would continue to decrease with decrease in species richness and local extinctions.

The species compositional changes induced by Lantana invasion are primarily driven by gradual changes in species density (Gooden et al. 2009). In this study, the density of tree seedling, juveniles and herbaceous vegetation reduced significantly $(\mathrm{p}<0.001)$ with increase in Lantana density. Also, the frequency of occurrence of some tree species decreased in LI sites than UI sites. However, the density of shrub and lianas was greater in LI sites compared to UI sites, due to higher L. camara density. Overall, Lantana invasion suppresses the growth of trees by impacting the younger stages of development, viz. the sapling and juvenile stages, whereas the adult trees remained largely unaffected. This proves that Lantana is unlikely to displace tree individuals that have attained adult stage and the resistance/susceptibility of a particular species to invasion (Gentle and Duggin 1997). For example, if a species is mainly represented by saplings in a particular location, it is easily susceptible and might eventually be displaced, but if it contains several adult individuals, it would resist invasion and is unlikely to get displaced (Gooden et al. 2009). Such variations in species density due to invasion by Lantana gradually alter landscape-level heterogeneity (Vitousek et al. 1996).

Invasion by Lantana has significantly $(\mathrm{p}<0.001)$ lowered the basal area of tree saplings, juveniles and herbs in $\mathrm{LI}$ sites compared to UI sites. This might be due to the formation of a thick Lantana thickets that alters the microenvironment (such as light and temperature), inhibiting either germination or growth of other species (Sharma and Raghubanshi 2007), thus lowering the basal area of tree saplings, juveniles and herbs. Furthermore, at the ground level, there occurs accumulation of litter of Lantana comprising of fallen leaves and dry debris which also causes allelopathic suppression of growth and recruitment of seedlings, juveniles and herbs (Gentle and Duggin 1997). Basal area acts as an indicator of growth and biomass (Lewis et al. 2004). The changes in quantitative ecological parameters such as density, basal area and IVI by invasion of Lantana leads to alteration in plant assemblage patterns and forest structural characteristics (Rusterholz et al. 2018). This may eventually create demographic instability among the species and reduce diversity in the future (Sharma and Raghubanshi 2010).

Analysis of tree size class distributions reveals the population structure of a forest (Newbery and Gartlan 1996). The composition and density distribution of saplings and juveniles indicate the future structure of the forest (Myo et al. 2016). A population structure comprising of an ample number of saplings and juveniles indicates good regeneration behaviour, while their insufficient numbers denote poor regeneration (Saxena and Singh 1985). In this study, tree density declined significantly ( $<0.001)$ with increase in diameter class in LI sites than Ul sites (Fig. 8). The highest contribution to the tree density was by lower diameter class of tree individuals $(<3-20 \mathrm{~cm}, 81.8 \%)$. This trend indicates that the tree species in the studied sites possess a good regenerative capacity. Even so, as observed in this study, tree saplings and tree juveniles were the most impacted life forms by Lantana invasion and further expansion of this species could affect their regenerative potential. Lantana is also known to displace natural scrub communities and prevent natural regeneration of some tree species (Ambika et al. 2003; Sharma and Raghubanshi 2006; Dobhal 2010). The recruitment of small-sized tree individuals into larger diameter classes has been known to be significantly impeded by Lantana invasion (Murali and Setty 2001; Sundaram and Hiremath 2012; Alemu and Terefe 2015). Although both UI and $\mathrm{LI}$ sites showed the same trend, UI sites had a higher number of small-sized tree individuals than the corresponding LI sites. This indicates that regenerative potential decreased post Lantana invasion.

The impacts of Lantana invasion were also reflected in the soil physico-chemical properties of the studied sites. While soil bulk density and pH were significantly $(p<0.001)$ lower in LI sites than UI sites, soil moisture, soil organic carbon and soil total nitrogen showed a significant ( $<<0.001)$ opposite trend. The observed tend is consistent with the findings of Niu et al. (2007), Dogra et al. (2009), Kumar et al. (2020). Invaded habitats experience changes in plant species composition and community structure and this alteration leads to changes in soil physico-chemical properties and nutrient dynamics (Hartman and McCarthy 2008; Rusterholz et al. 2018). The alteration in soil properties could be due to the release of allelochemicals by Lantana. The allelochemicals

Page $14 / 25$ 
released by the invasive plant species is known to alter the soil physical and chemical properties and regulate the biotic communities (Nardi et al. 2000). Very often, the altered soil properties provide favourable conditions for further invasion by Lantana and other invasive species (Niu et al. 2007). Soil moisture, soil organic carbon and total nitrogen are known to promote invasive species growth (Kumar et al. 2020). Therefore, invasion by Lantana not only affects the native resident plant community structure and composition, but also changes soil physio-chemical properties that may not be suitable for their growth.

In the present study, species richness, density, basal area, diversity indices (Shannon, Simpson, evenness, Fisher's alpha and Margalef) of tree seedlings, juveniles and herbs showed a significant negative correlation with Lantana densities (Table 4). Whereas, negative non-significant correlation with tree adults and total species (Table 4). Soil organic carbon (SOC), soil total nitrogen (STN) and soil moisture (M\%) showed a significant positive correlation with Lantana densities, whereas, a significant negative correlation has been observed between pH and soil bulk density with Lantana density(Table 4). Similar, findings has been recorded by Badalamenti et al. (2016) in Mediterranean ecosystems of Linosa island. In this study, Lantana density is found to be significantly positively correlated with its own biomass ( $\leq \leq 0.01)$, and shrub and liana density ( $\leq 0.01)$, which could be attributed to its dominance in the shrub and liana category. Invasive plants are known to alter the soil pools and processes in ecosystems that they invade in by changing the quantity, quality, decomposition and mineralization (Ashton et al. 2005; Ruwanza and Shackleton 2016). Lantana density increases litter inputs and its chemical composition is also different from the native forest litter. The Lantana litter is rich in high SOC, $\mathrm{N}$ and soil moisture with low lignin content and favourable microclimate having faster decomposition rate and release of $\mathrm{N}$. These alterations beneath the Lantana canopy significantly alter the SOC, total nitrogen pools and soil moisture in soils (Sharma and Raghubanshi 2009) and making the soil properties ideal for Lantana growth. The significantly higher M (\%) content in LI sites than the UI sites could be due to the reason of soil water repellency due to increase in debris and organic matter from dead Lantana leaves, which are known to decompose slowly (Fan et al. 2010; Singh et al. 2014). Lantana produce a substantial quantity of litter (Singh et al. 2014) rich in allelopathic chemicals (El-Kenany and ElDarier 2013) which might react with organic matter and hydrophobic compounds to trigger repellency in the soils (Ruwanza et al. 2013). Soil bulk density and pH were significantly lower in LI sites than UI sites, which could be due the higher litter content and its decomposition by micro-organisms. Similary results have been observed by Sharma and Raghubanshi (2009) in tropical dry deciduous forests of India. This could be the reason of Lantana success as an invasive species in these ecosystems. Changes in soil nutrients following Lantana invasion could be a contributor to its successful proliferation. Levine et al. (2006) also opined that such increase in nutrients positively affect the growth and spread of the invader in the form of the "push and pull" theory of invasion.

\section{Conclusions}

The present study revealed a significant negative impact of an alien invasive shrub, Lantana on species richness, density, basal area and diversity of vegetation of native plant communities. It is clear that Lantana-invaded sites comprise significantly lower richness, density and basal area than uninvaded sites. It not only impacts the vegetation of native communities, but also alters the soil properties, conclusively by favouring its growth. Furthermore, the alterations in vegetation structure, composition and soil properties could lead to changes in other tropical levels and change ecosystem functioning. Appropriate methods and long-term monitoring studies in permanent plots are needed for better understanding, management, and restoration as an effective and efficient conservation strategy for invaded landscapes in tropical forests.

\section{Declarations}

\section{Acknowledgements}

We are thankful to the Madhya Pradesh State Forest Department and Forest Department of Sagar district for permission and for providing the necessary facilities and staff support during the field work. We also thank Prof. Pramod Kumar Khare, Department of Botany for the identification of plant specimens.

\section{Author's contributions}

The study was conceptualized and designed by Parvaiz A Lone, Javid A Dar, Subashree K and Mohammed L Khan. Material preparation, field work, data collection and analysis were performed by Parvaiz A Lone, Javid A Dar and Subashree. The first draft of the manuscript was written by Parvaiz A Lone, Javid A Dar and Subashree providing review and comments by Mohammed L Khan. All authors's read and approved the submitted version of the manuscript.

\section{Funding}

This study was supported by Science and Engineering Research Board (SERB), Department of Science and Technology, New Delhi under National PostDoctoral Fellowship Scheme (Ref. No.: PDF/2015/000447) to the second author. We would also like to thank the Department of Biotechnology, Government of India (No. BT/PR12899/ NDB/39/506/2015 dt. 20/06/2017) for partial funding

\section{Conflicts of interest}

No potential conflict of interest was reported by the authors

\section{Availability of data and material}

The data that support the findings of this study are available on request from the corresponding author

\section{Abbreviations}




\begin{tabular}{|c|c|}
\hline SR & species Richness \\
\hline $\mathrm{DBH}$ & diameter at breast height \\
\hline soc & soil organic carbon \\
\hline STN & soil total nitrogen \\
\hline M & soil moisture \\
\hline $\mathrm{m}$ & Meter \\
\hline $\mathrm{N}$ & North \\
\hline $\mathrm{E}$ & East \\
\hline${ }^{\circ} \mathrm{C}$ & degree Celsius \\
\hline D & density \\
\hline BA & basal area \\
\hline IVI & Importance Value Index \\
\hline APG & Angiosperm Phylogeny Group \\
\hline GRIN & Germplasm Resources Information Network \\
\hline C & carbon \\
\hline $\mathrm{cm}$ & centimeters \\
\hline $\mathrm{H} \rrbracket$ & Shannon index \\
\hline $\mathrm{Cd}$ & Simpson's dominance index \\
\hline$E$ & evenness \\
\hline $\mathrm{R}$ & Margalef's index \\
\hline ANOVA & analysis of variance \\
\hline
\end{tabular}

\section{References}

1. Ahmad R, Khuroo AA, Hamid M, Rashid I (2019) Plant invasion alters the physico-chemical dynamics of soil system: insights from invasive Leucanthemum vulgare in the Indian Himalaya. Environ Monit Assess 191:792. https://doi.org/10.1007/s10661-019-7683-x

2. Alemu SC, Terefe AA (2015) Impact of invasion: A case study on the ecological and socioeconomic impact of Lantana camara (L.) in Abay Millennium Park (AMP), Bahir Dar, Ethiopia. J Ecol Nat 7(5):132-145

3. Ambika SR, Poornima S, Palaniraj R, Sati SC, Narwal SS (2003) Allelopathic plants. 10. Lantana camara L. Allelopathy J 12(2):147-162

4. Angiosperm Phylogeny Group IV (APG IV), http://www.mobot.org/MOBOT/research/APweb/ 2019 Last accessed on 16/05/2019

5. Ashton IW, Hyatt LA, Howe KM, Gurevitch J, Lerdau MT (2005) Invasive species accelerate decomposition and litter nitrogen loss in a mixed deciduous forest. Ecol Appl 15(4):1263-1272

6. Badalamenti E, Gristina L, Laudicina VA, Novara A, Pasta S, Mantia TL (2016) The impact of Carpobrotus cfr. Acinaciformis (L.) L. Bolus on soil nutrients, microbial communities structure and native plant communities in Mediterranean ecosystems. Plant Soil 409:19-34. DOI 10.1007/s11104-016-2924-z

7. Barney JN, Tekiela DR, Barrios-Garcia MN, Dimarco RD, Hufbauer RA, Leipzig-Scott P, Nuñez MA, Pauchard A, Pyšek P, Vítková M, Maxwell BD (2015) Global Invader Impact Network (GIIN): toward standardized evaluation of the ecological impacts of invasive plants. Ecol Evol 5(14):2878-2889

8. Bellard C, Cassey P, Blackburn TM (2016) Alien species as a driver of recent extinctions. Biol Lett 12:20150623. http://dx.doi.org/10.1098/rsbl.2015.0623

9. Brown KA, Scatena FN, Gurevitch J (2006) Effects of an invasive tree on community structure and diversity in a tropical forest in Puerto Rico. For Ecol Manag 226:145-152

10. Cabin RJ, Weller SG, Lorence DH, Cordell S, Hadway LJ, Montgomery R, Goo D, Ukakami A (2002) Effects of light, alien grass, and native species additions on Hawaiian dry forest restoration. Ecolo Appl 12(6):1595-1610

11. Catford JA, Daehler CC, Murphy HT, Sheppard AW, Hardesty BD, Westcott DA, Rejmánek M, Bellingham PJ, Pergl J, Horvitz CC, Hulme PE (2012) The intermediate disturbance hypothesis and plant invasions: Implications for species richness and management. Perspect Plant Ecol Evol Syst 14:231-241

12. Champion HG, Seth SK (1968) A revised survey of the forest types of India. Govt. of India Press, New Delhi, p 404

13. Curtis JT, Mclntosh RP (1951) An upland forest continuum in the prairie-forest border region of Wisconsin. Ecology 32(3):476-496. doi:10.2307/1931725 
14. D'Antonio CM, Vitousek PM (1992) Biological invasions by exotic grasses, the grass/fire cycle, and global change. Annu Rev Ecol Systemat 23(1):63-87

15. Dar JA, Subashree K, Sundarapandian S, Saikia P, Kumar A, Khare PK, Dayanandan S, Khan ML (2019) Invasive species and their impact on tropical forests of Central India: A Review. In: Tropical Ecosystems: Structure, Functions and Challenges in the Face of Global Change. Springer, Singapore, pp 69109

16. Dassonville N, Vanderhoeven S, Vanparys V, Hayez M, Gruber W, Meerts P (2008) Impacts of alien invasive plants on soil nutrients are correlated with initial site conditions in NW Europe. Oecologia 157:131-140

17. Day MD, Wiley CJ, Playford J, Zalucki MP (2003) Lantana: current management status and future prospects. ACIAR, Canberra

18. Dobhal PK, Kohli RK, Batish DR (2010) Evaluation of the impact of Lantana camara L. invasion, on four major woody shrubs, along Nayar river of Pauri Garhwal in Uttarakhand Himalaya. Int J Biodivers Conserv 2:155-161

19. Dobhal PK, Kohli RK, Batish DR (2011) Impact of Lantana camara L. invasion on riparian vegetation of Nayar region in Garhwal Himalayas. J Ecol Nat Environ 3:11-22

20. Dogra KS, Kohli RK, Sood SK (2009) An assessment and impacts of three invasive species in the Shivalik hills of Himachal Pradesh, India. Int J Biodivers Conserv 1(1):004-010

21. Ehrenfeld JG (2003) Effects of exotic plant invasions on soil nutrient cycling processes. Ecosystems 6:503-523

22. Ehrenfeld JG (2010) Ecosystem consequences of biological invasions. Annu Rev Ecol Evol Syst 41:59-80. 10.1146/annurev-ecolsys-102209-144650

23. El-Kenany ET, El-Darier SM (2013) Suppression effects of Lantana camara L. aqueous extracts on germination efficiency of Phalaris minor Retz. and Sorghum bicolor L. (Moench). J Taibah Univ Sci 7:64-71

24. Fan L, Chen Y, Yuan J, Yang Z (2010) The effect of Lantana camara Linn. invasion on soil chemical and microbiological properties and plant biomass accumulation in southern China. Geoderma 154:370-378

25. Fei S, Phillips J, Shouse M (2014) Biogeomorphic impacts of invasive species. Annu Rev Ecol Evol Syst 45:69-87

26. Gaertner M, Biggs R, te Beest M, Hui C, Molofsky J, Richardson DM (2014) Invasive plants as drivers of regime shifts: identifying high priority invaders that alter feedback relationships. Divers Distrib 20:733-744

27. Gentle CB, Duggin JA (1997) Allelopathy as a competitive strategy in persistent thickets of Lantana camara L. in three Australian forest communities. Plant Ecol 132:85-95

28. Global Invasive Species Database (2020) Species profile: Lantana camara. Downloaded from http://www.iucngisd.org/gisd/species.php?sc=56 on 28-122020

29. Gooden B, French K, Turner PJ, Downey PO (2009) Impact threshold for an alien plant invader, Lantana camara L., on native plant communities. Biol Conserv 142:2631-2641

30. Goyal N, Esler KJ, Sharma GP (2018) What drives performance potential of Lantana camara L. (sensu lato) in the invaded range? Trop Ecol 59(1):57-68

31. Hammer $\varnothing$, Harper DAT, Ryan PD. Past (2001) Paleontological Statistics Software Package for Education and Data Analysis. Palaeont Electron 4:4-9

32. Hartman KM, McCarthy BC (2008) Changes in forest structure and species composition following invasion by a non-indigenous shrub, Amur honeysuckle (Lonicera maackii). J Torrey Bot Soc 245-259. doi:10.3159/TORREY-D-15-00036.1

33. Hejda M, Pysek P (2006) What is the impact of Impatiens glandulifera on species diversity of invaded riparian vegetation? Biol Conserv 132:143-152

34. Hejda M, Pysek P, Jarosík V (2009) Impact of invasive plants on the species richness, diversity and composition of invaded communities. J Ecol 97:393403

35. Hellmann JJ, Byers JE, Bierwagen BG, Dukes JS (2008) Five potential consequences of climate change for invasive species. Conserv Biol 22:534-543

36. Janzen DH (1988) Tropical dry forests: the most endangered major tropical ecosystem. In: Wilson EO (ed) Biodiversity. National Academic Press, Washington, DC, pp 130-137

37. Johnstone IM (1986) Plant invasion windows: a time based classification of invasion potential. Biol Rev 61:369-394

38. Kannan R, Shackleton CM, Shaanker RU (2013) Reconstructing the history of introduction and spread of the invasive species, Lantana, at three spatial scales in India. Biol Invasions 15(6):1287-1302

39. Kershaw KA (1964) Quantitive and Dynamic Ecology. Edward Arnold (Publishers) Ltd., London, p 308

40. Kirk PL (1950) Kjeldahl Method for Total Nitrogen. Anal Chem 22(2):354-358. https://doi.org/10.1021/ac60038a038

41. Kohli RK, Batish DR, Singh HP, Dogra KS (2006) Status, invasiveness and environmental threats of three tropical American invasive weeds (Parthenium hysterophorus L., Ageratum conyzoides L., Lantana camara L.) in India. Biol Invasions, 8(7): 1501-1510

42. Kumar M, Verma AK, Garkoti SC (2020) Lantana camara and Ageratina adenophora invasion alter the understory species composition and diversity of chir pine forest in central Himalaya, India. Acta Oecol. https://doi.org/10.1016/j.actao.2020.103642

43. Lazzaro L, Bolpagni R, Buffa G, Gentili R, Lonati M, Stinca A, Acosta ATR, Adorni M, Aleffi M, Allegrezza M, Angiolini C, Assini S, Bagella S, Bonari G, Bovio M, Bracco F, Brundu G, Caccianiga M, Carnevali L, Di cecco V, Ceschin S, Ciaschetti G, Cogoni A, Foggi B, Frattaroli AR, Genovesi P, Gigante D, Lucchese F, Mainetti A, Mariotti M, Minissale P, Paura B, Pellizzari M, Perrino EV, Pirone G, Poggio L, Poldini L, Poponessi S, Prisco I, Prosser F, Puglisi M, Rosati L, Selvaggi A, Sottovia L, Spampinato G, Stanisci A, Venanzoni R, Viciani D, Vidali M, Villani M, Lastrucci L (2020) Impact of invasive alien plants on native plant communities and Natura 2000 habitats: State of the art, gap analysis and perspectives in Italy. J Environ Manage 274:111140. doi:10.1016/j.jenvman.2020.111140

44. Levine JM, Pachepsky E, Kendall BE, Yelenik SG, Lambers JHR (2006) Plant-soil feedbacks and invasive spread. Ecol Lett 9:1005-1014 
45. Levine JM, Vilà M, D’Antonio CM, Dukes JS, Grigulis K, Lavorel S (2003) Mechanisms underlying the impacts of exotic plant invasions. Proc R Soc Lond B 270:775-781

46. Lewis SL, Phillips OL, Baker TR, Lloyd J, Malhi Y, Almeida S, Higuchi N, Laurance WF, Neill DA, Silva JN, Terborgh J, Lezama AT, Martinez RV, Brown S, Chave J, Kuebler C, Vargas PN, Vinceti B (2004) Concerted changes in tropical forest structure and dynamics, evidence from 50 South American long-term plots. Philos Trans R Soc B Biol Sci 359:421-436. https://doi.org/10.1098/rstb.2003.1431

47. Liao C, Peng R, Luo Y, Zhou X, Wu X, Fang C, Chen J, Li B (2008) Altered ecosystem carbon and nitrogen cycles by plant invasion: a meta-analysis. New Phytol 177(3):706-714

48. Lone PA, Dar JA, Subashree K, Raha D, Pandey PK, Ray T, Khare PK, Khan ML (2019) Impact of plant invasion on physical, chemical and biological aspects of ecosystems: A review. Trop Plant Res 6(3):528-544

49. Lowe S, Browne M, Boudjelas S, De Poorter M (2000) 100 of the world's worst invasive alien species. A selection from the Global Invasive Species Database. Invasive Species Specialist Group (ISSG) a specialist group of the Species Survival Commission (SSC) of the World Conservation Union (IUCN)

50. Misra R (1968) Ecology Work Book. Oxford and IBH Publishing Co., New Delhi, p 244

51. Mungi NA, Qureshi Q, Jhala YV (2020) Expanding niche and degrading forests: Key to the successful global invasion of Lantana camara (sensu lato). Glob Ecol Conserv 23:e01080

52. Murali KS, Setty RS (2001) Effect of weeds: Lantana camara and Chromelina odorata growth on the species diversity, regeneration and stem density of tree and shrub layer in BRT Sanctuary, India. Curr Sci 80:675-678

53. Myo KK, Thwin S, Khaing N (2016) Floristic composition, structure and soil properties of mixed deciduous forest and deciduous dipterocarp forest: Case study in Madan watershed, Myanmar. Am J Plant Sci 7:279-287

54. Nardi S, Concheri G, Pizzeghello D, Sturaro A, Rella R, Parvoli G (2000) Soil organic matter mobilization by root exudates. Chemosphere 5:653-658

55. Newbery DM, Gartlan JS (1996) A structural analysis of rain forest at Korup and Douala-Edea, Cameroon. Proc R Soc Edinb Sec B Biol Sci 104:177$22410.1017 /$ S0269727000006138

56. Niu HB, Liu WX, Wan FH, Liu B (2007) An invasive aster (Ageratina adenophora) invades and dominates forest understories in China: altered soil microbial communities facilitate the invader and inhibit natives. Plant Soil 294:73-85. doi:10.1007/s11104-007-9230-8

57. Oommachan M (1977) The flora of Bhopal (angiosperms). JK Jain Brothers, Bhopal, p 475

58. Panda PC, Mahapatra AK, Acharya PK, Debata AK (2013) Plant diversity in tropical deciduous forests of Eastern Ghats, India, A landscape level assessment. Int J Biodivers Conserv 5:625-639. https://doi.org/10.5897/IJBC2013.0581x

59. Pearson T, Walker S, Brown S (2005) Sourcebook for land use, land-use change and forestry projects. Winrock International and the BioCarbon Fund of the World Bank

60. Potgieter LJ, Strasberg D, Wilson JRU, Richardson DM (2014) Casuarina invasion alters primary succession on lava flows in the Mascarene Islands. Biotropica 46:268-275

61. Pysek P, Jarošík V, Hulme PE, Pergl J, Hejda M, Schaffner U, Vilà M (2012) A global assessment of invasive plant impacts on resident species, communities and ecosystems: the interaction of impact measures, invading species' traits and environment. Glo Chan Biol 18(5):1725-1737

62. Pyšek P, Pyšek A (1995) Invasion by Heracleum mantegazzianum in different habitats in the Czech Republic. J Veg Sci 6(5):711-718. doi: $10.2307 / 3236442$

63. Raghubanshi AS, Tripathi A (2009) Effect of disturbance, habitat fragmentation and alien invasive plants on floral diversity in dry tropical forests of Vindhyan highland: a review. Trop Ecol 50(1):57-69

64. Ramírez-Albores JE, Badano El, Flores J, Flores-Flores JL, Yáñez-Espinosa L (2019) Scientific literature on invasive alien species in a megadiverse country: advances and challenges in Mexico. NeoBiota 48:113

65. Rejmánek M (1995) What makes a species invasive?. - In: P. Pysek et al (eds), Plant invasions. SPB Academic Publ, pp 3-13

66. Richardson DM, Macdonald IA, Forsyth GC (1989) Reduction in plant species richness under stands of alien trees and shrubs in fynbos biome. South Afr For J 149:1-8

67. Richardson DM, Rejmanek M (2011) Trees and shrubs as invasive alien species-a global review. Divers Distrib 17(5):788-809

68. Rusterholz HP, Schneuwly J, Baur B (2018) Invasion of the alien shrub Prunus laurocerasus in suburban deciduous forests: effects on native vegetation and soil properties. Acta Oecol 92:44-51. doi:10.1016/j.actao.2018.08.004

69. Ruwanza S, Gaertner M, Richardson DM, Esler KJ (2013) Soil water repellency in riparian systems invaded by Eucalyptus camaldulensis: a restoration perspective from the Western Cape Province, South Africa. Geoderma 200-201:9-17

70. Ruwanza S, Shackleton CM (2016) Effects of the invasive shrub, Lantana camara, on soil properties in the Eastern Cape, South Africa. Weed Biol Manag 16(2):67-79. doi:10.1111/wbm.12094

71. Saxena AK, Singh JS (1985) Tree population structure of certain Himalayan forest association and implications concerning their future composition. Vegetatio 58:61-69

72. Seebens H, Blackburn TM, Dyer EE, Genovesi P, Hulme PE, Jeschke JM, Pagad S, Pyšek P, Winter M, Arianoutsou M, Bacher S, Blasius B, Brundu G, Capinha C, Celesti-Grapow L, Dawson W, Dullinger S, Fuentes N, Jäger H, Kartesz J, Kenis M, Kreft H, Kühn I, Lenzner B, Liebhold A, Mosena A, Moser D, Nishino M, Pearman D, Pergl J, Rabitsch W, Rojas-Sandoval J, Roques A, Rorke S, Rossinelli S, Roy HE, Scalera R, Schindler S, Štajerová K, Tokarska-Guzik B, van Kleunen M, Walker K, Weigelt W, Yamanaka T, EssI F (2017) No saturation in the accumulation of alien species worldwide. Nat Commun 8:14435. doi:10.1038/ncomms14435 
73. Sharma GP, Raghubanshi AS (2010) How Lantana invades dry deciduous forest: a case study from Vindhyan highlands, India. Trop Ecol 51(2):305-316

74. Sharma GP, Raghubanshi AS (2006) Tree population structure, regeneration and expected future composition at different levels of Lantana camara L. invasion in the Vindhyan tropical dry deciduous forest of India. Lyonia 11(1):27-39

75. Sharma GP, Raghubanshi AS (2007) Effect of Lantana camara L. cover on local depletion of tree population in the Vindhyan tropical dry deciduous forest of India. Appl Ecol Env Res 5(1):109-121

76. Sharma GP, Raghubanshi AS (2009) Lantana invasion alters soil nitrogen pools and processes in the tropical dry deciduous forest of India. Appl Soil Ecol 42(2):134-140

77. Sharma GP, Raghubanshi AS, Singh JS (2005) Lantana invasion: an overview. Weed Biol Manag 5(4):157-165

78. Simberloff D, Louis Martin J, Genovesi P, Maris V, Wardle DA, Aronson J, Courchamp F, Galil B, García-Berthou E, Pascal M, Pyšek P, Sousa R, Tabacchi E, Vilà M (2013) Impacts of biological invasions: what's what and the way forward. Trends Ecol Evol 28(1):58-66

79. Singh HP, Batish DR, Dogra KS, Kaur S, Kohli RK, Negi A (2014) Negative effect of litter of invasive weed Lantana camara on structure and composition of vegetation in the lower Siwalik Hills, northern India. Environ Monit Assess 186:3379-3389

80. Stevens PF (2017) Angiosperm Phylogeny Website. Version 14. http://www.mobot.org/MOBOT/research/APweb/

81. Stinson K, Kaufman S, Durbin L, Lowenstein F (2007) Impacts of garlic mustard invasion on a forest understory community. Northeast Nat 14(1):73-88

82. Subashree K, Dar JA, Karuppusamy S, Sundarapandian SM (2020) Plant diversity, structure and regeneration potential in tropical forests of Western Ghats, India. Acta Ecol Sin https://doi.org/10.1016/j.chnaes.2020.02.004

83. Sundaram B, Hiremath AJ (2012) Lantana camara invasion in a heterogeneous landscape: patterns of spread and correlation with changes in native vegetation. Biol Invasions 14:1127-1141. DOI 10.1007/s10530-011-0144-2

84. Taylor S, Kumar L, Reid N, Kriticos DJ (2012) Climate change and the potential distribution of an invasive shrub, Lantana camara L. PLoS ONE 7(4):e35565. doi:10.1371/journal.pone.0035565

85. Timsina B, Shrestha BB, Rokaya MB, Münzbergová Z (2011) Impact of Parthenium hysterophorus L. invasion on plant species composition and soil properties of grassland communities in Nepal. Flora 206:233-240

86. Truscott AM, Palmer SC, Soulsby C, Westaway S, Hulme PE (2008) Consequences of invasion by the alien plant Mimulus guttatus on the species composition and soil properties of riparian plant communities in Scotland. Perspect Plant Ecol Evol Syst 10(4):231-240

87. Veldman JW, Putz FE (2010) Long-distance dispersal of invasive grasses by logging vehicles in a tropical dry forest. Biotropica 42(6):697-703. 10.1111/j.1744-7429.2010.00647.x

88. Vilà M, Espinar JL, Hejda M, Hulme PE, Jarošík V, Maron JL, Pergl J, Schaffner U, Sun Y, Pyšek P (2011) Ecological impacts of invasive alien plants: a meta-analysis of their effects on species, communities and ecosystems. Ecol Lett 14:702-708

89. Vitousek PM (1990) Biological invasions and ecosystem processes. Towards an integration of population biology and ecosystem studies. Oikos 57:7-13

90. Vitousek PM (1994) Beyond global warming: ecology and global change. Ecology 75(7):1861-1876

91. Vitousek PM, D’Antonio CM, Loope LL, Westbrooks R (1996) Biological invasions as global environmental change. Am Sci 84:468

92. Walkley A, Black IA (1934) An examination of the Degtjareff method for determining soil organic matter, and a proposed modification of the chromic acid titration method. Soil Sci 37:29-38

93. Wiersema JH (2019) GRIN Taxonomy. US National Plant Germplasm System. Checklist dataset https://doi.org/10.15468/ao14pp accessed via GBIF.org on $2020-12-28$

94. Wilsey BJ, Potvin C (2000) Biodiversity and ecosystem functioning: importance of species evenness in an old field. Ecology 81:887-892

\section{Figures}




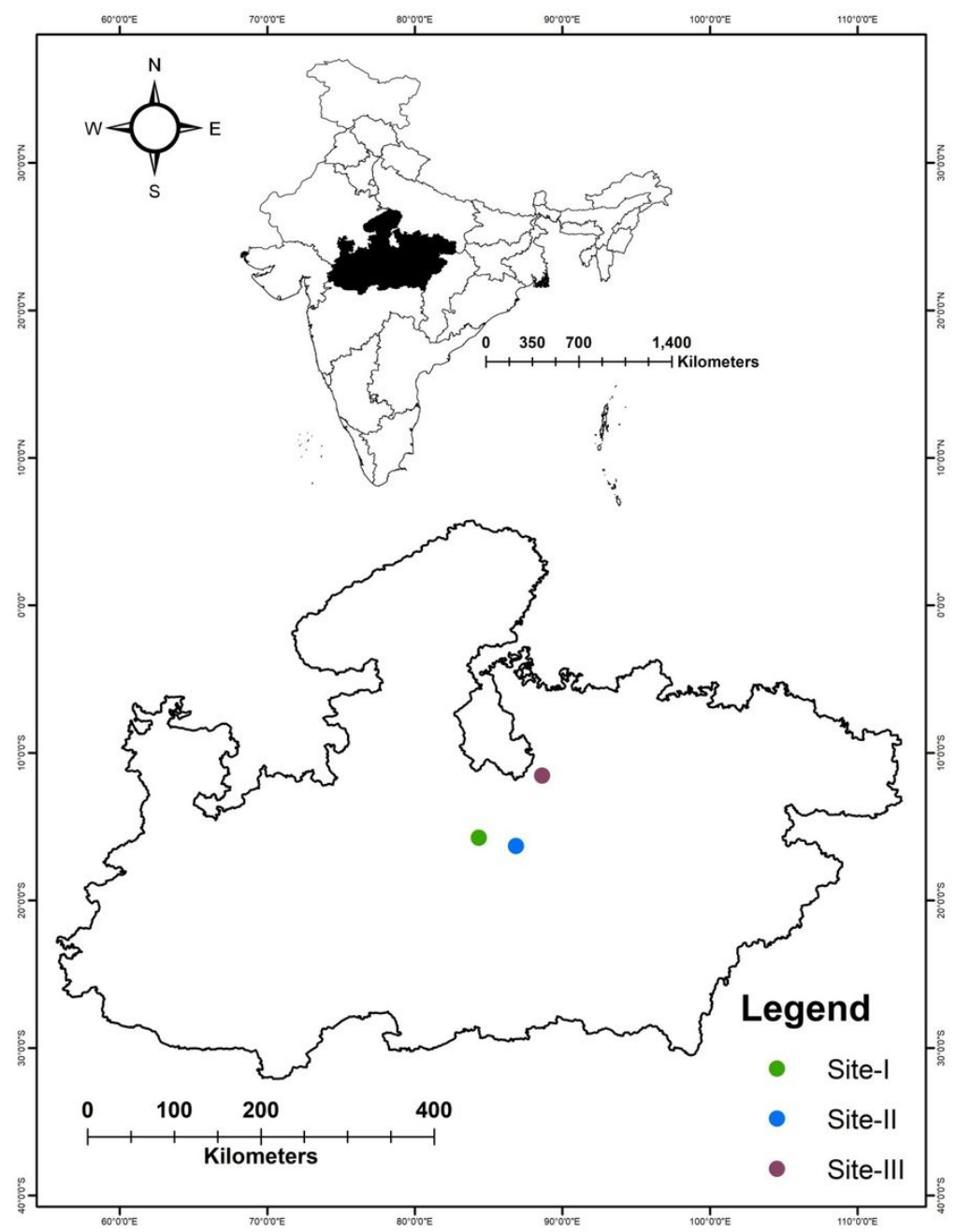

\section{Figure 1}

Geographical location of three forests study sites of Madhya Pradesh, Central India. Note: The designations employed and the presentation of the material on this map do not imply the expression of any opinion whatsoever on the part of Research Square concerning the legal status of any country, territory, city or area or of its authorities, or concerning the delimitation of its frontiers or boundaries. This map has been provided by the authors. 
(a)

"Change (\%) $=\mathrm{LI}=\mathrm{UI}$

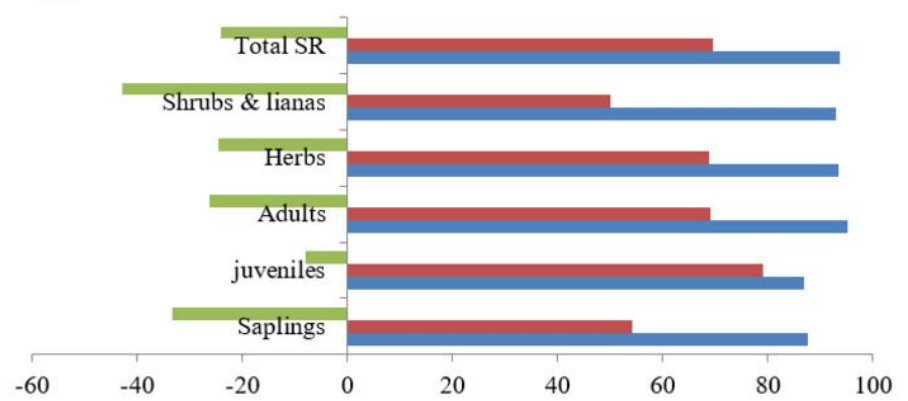

(b)
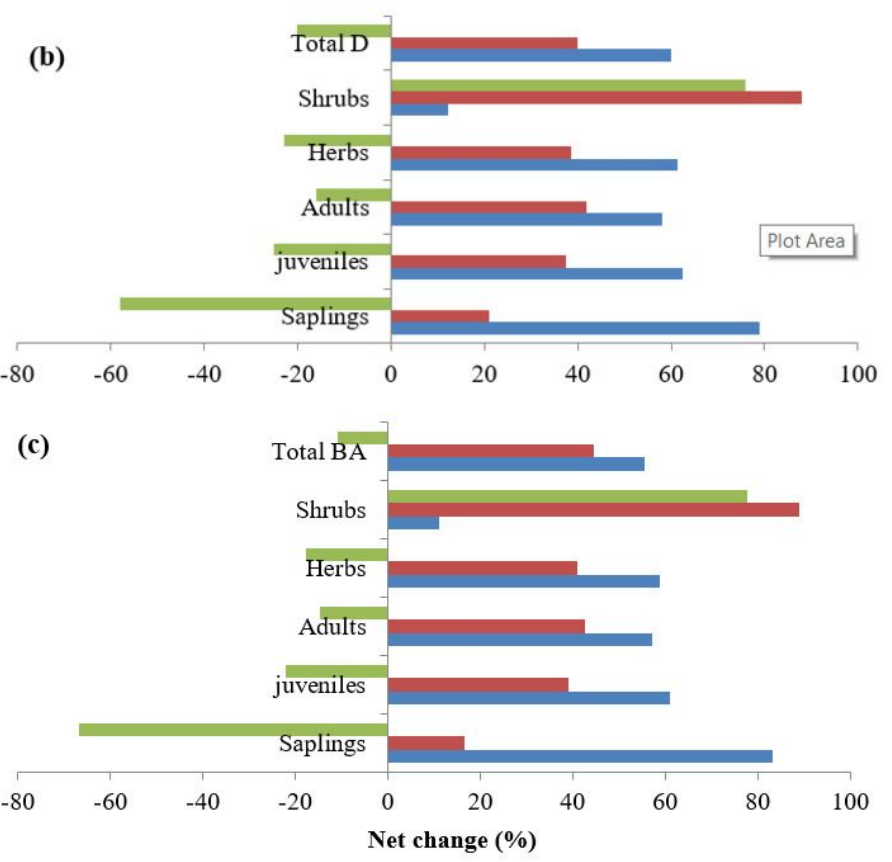

\section{Figure 2}

Percent (\%) change in a) species richness (SR), b) density (D) and c) basal area (BA) of saplings, juveniles, adults, herbs, shrubs \& lianas and total SR, in uninvaded (UI) and Lantana-invaded (LI) sites 

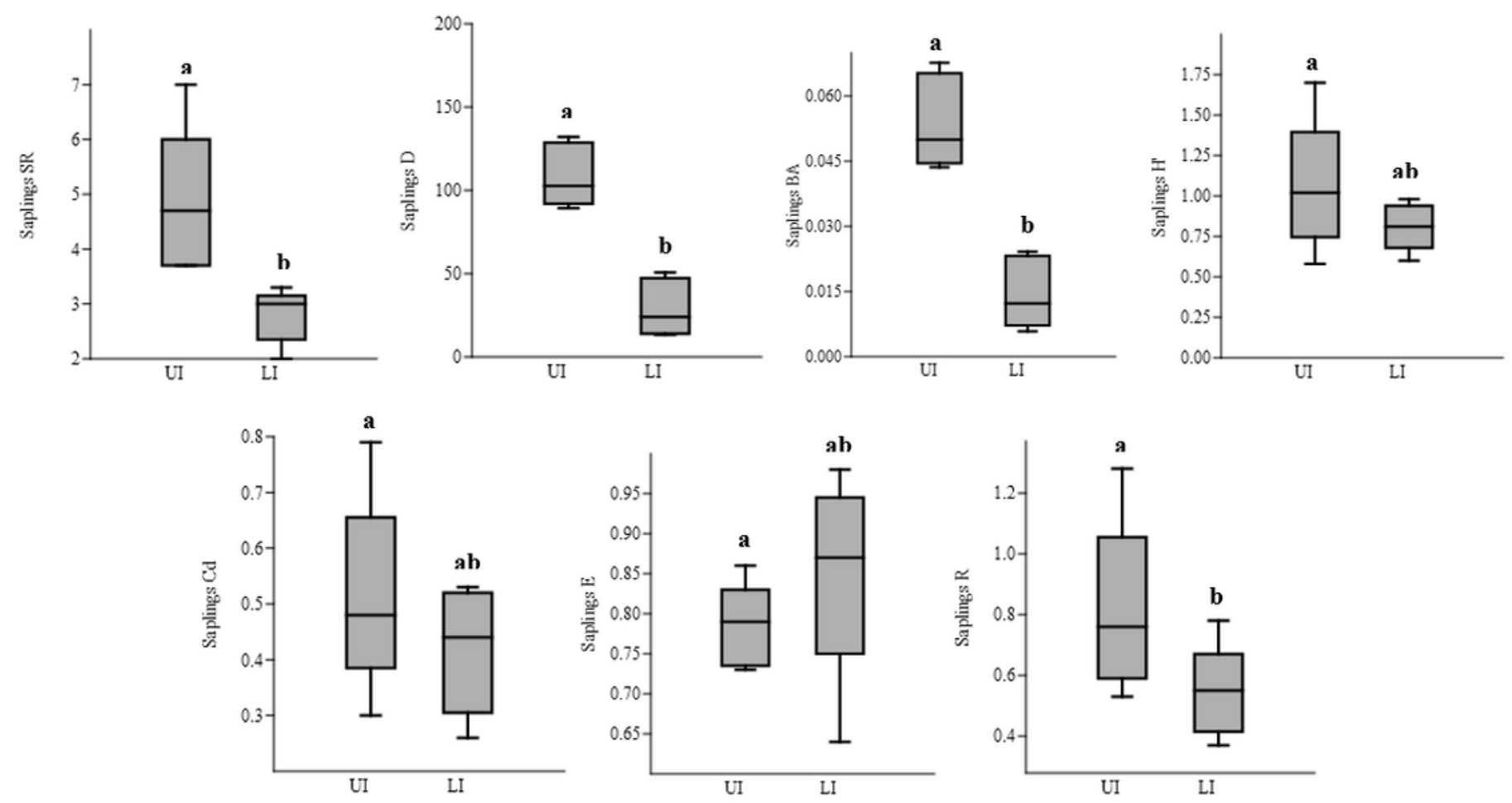

Figure 3

Species richness (SR; No. of species), density (D; stems ha-1), basal area (BA; m2 ha-1), Shannon index (H区), Simpson index (Cd), evenness index (E) and Margalef's index (R) of tree seedlings in uninvaded (UI) and Lantana-invaded (LI) sites. Data are presented as the mean value, different letters are significantly different at $\mathrm{P}<0.05$
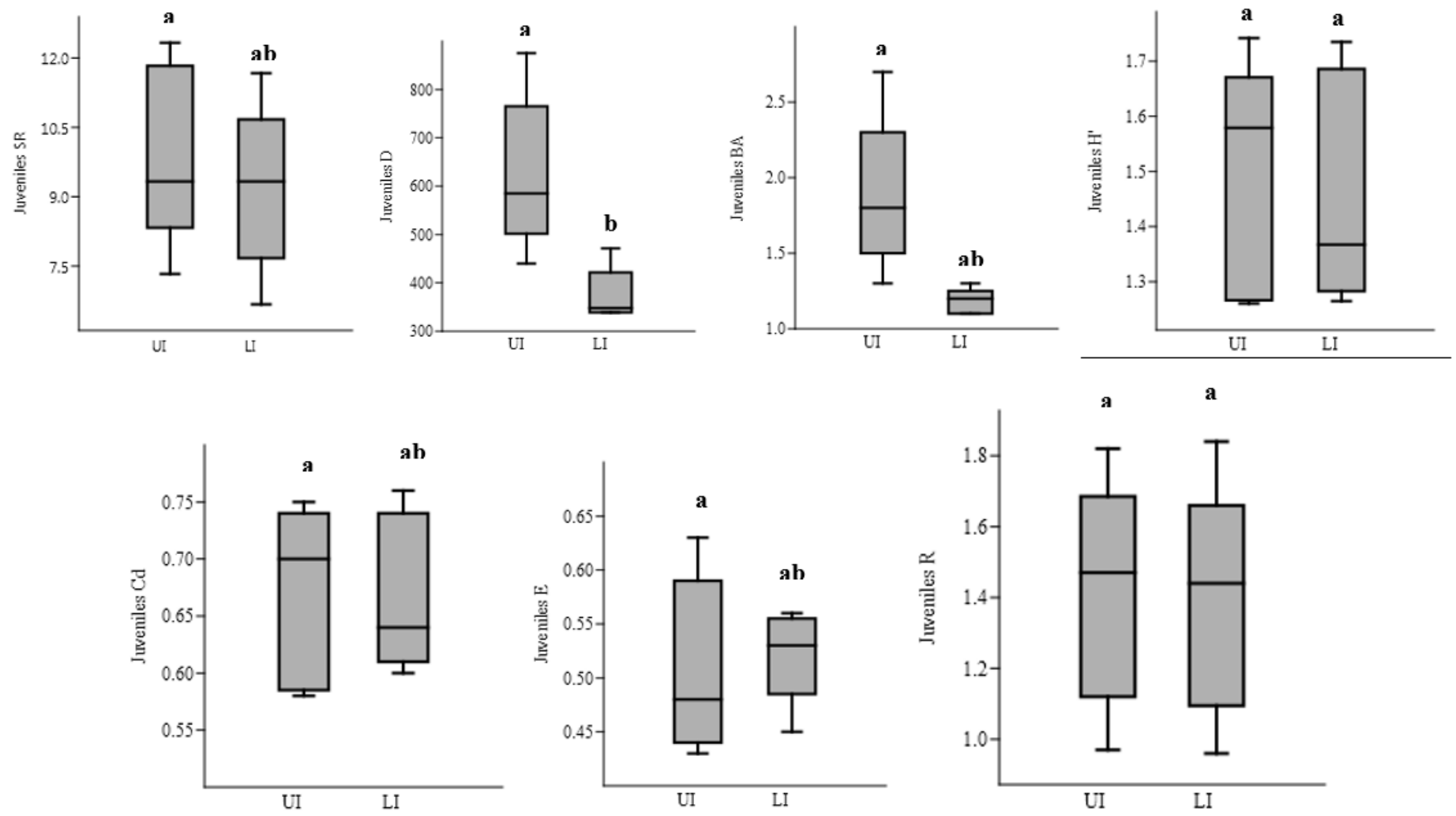

\section{Figure 4}

Species richness (SR; No. of species), density (D; stems ha-1), basal area (BA; m2 ha-1), Shannon index (HZ), Simpson index (Cd), evenness index (E) and Margalef's index (R) of tree juveniles in uninvaded (UI) and Lantana-invaded (LI) sites. Data are presented as the mean value, different letters are significantly 
different at $\mathrm{P}<0.05$
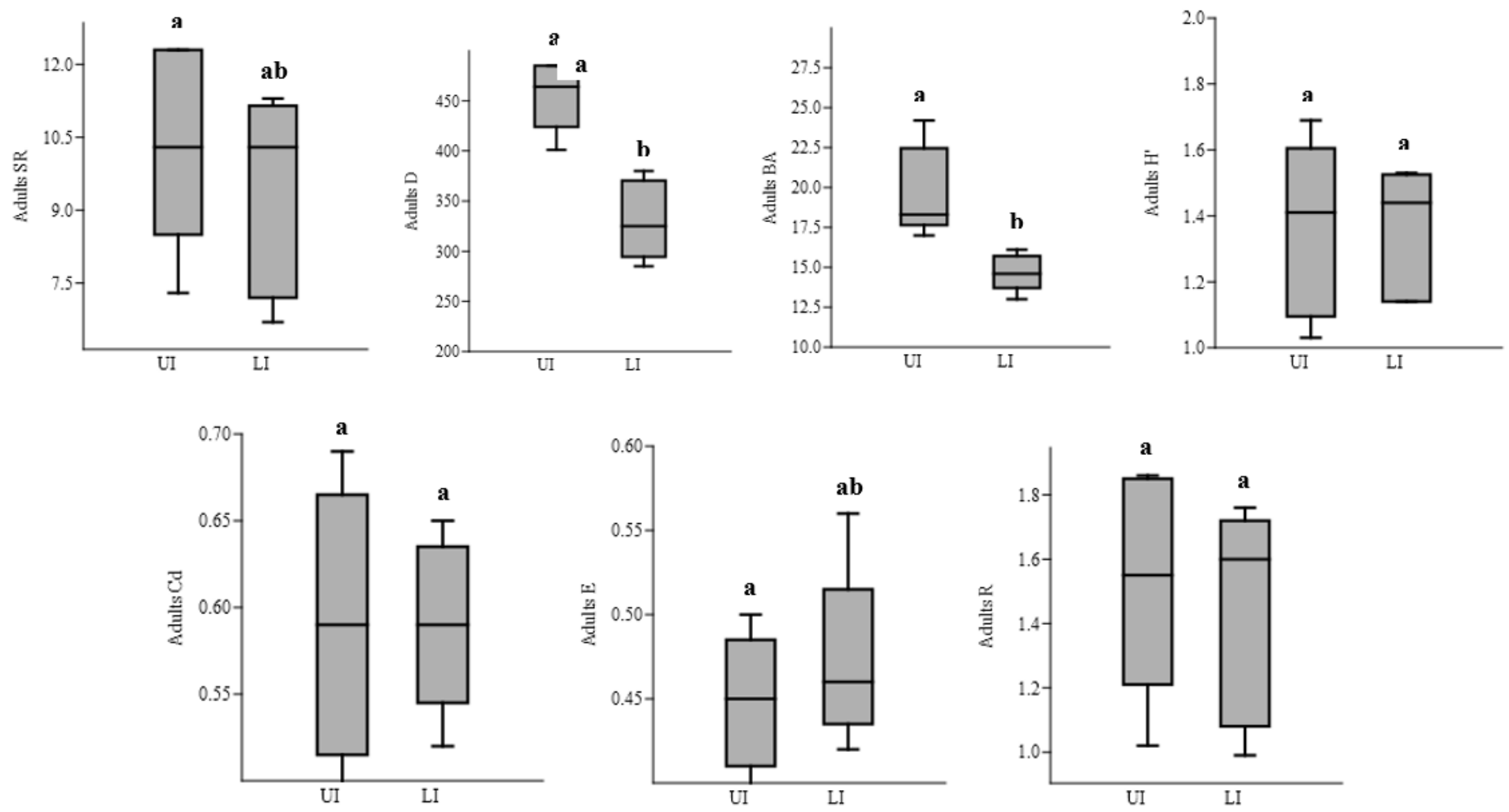

\section{Figure 5}

Species richness (SR; No. of species), density (D; stems ha-1), basal area (BA; m2 ha-1), Shannon index (H区), Simpson index (Cd), evenness index (E) and Margalef's index (R) of tree adults in uninvaded (UI) and Lantana-invaded (LI) sites. Data are presented as the mean value, different letters are significantly different at $\mathrm{P}<0.05$
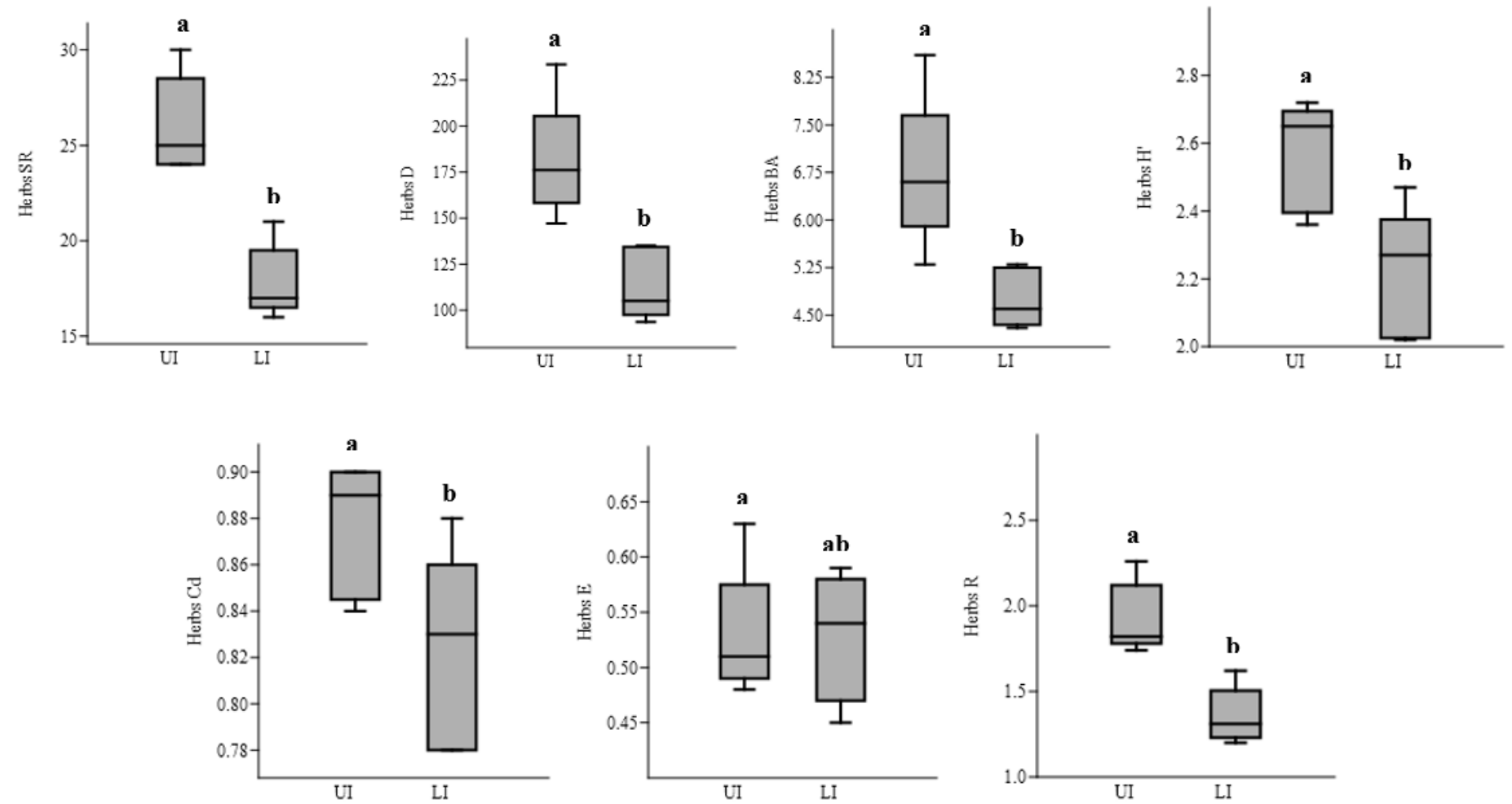

Figure 6 
Species richness (SR; No. of species), density (D; stems ha-1), basal area (BA; m2 ha-1), Shannon index (H区), Simpson index (Cd), evenness index (E) and Margalef's index (R) of herbs in uninvaded (UI) and Lantana-invaded (LI) sites. Data are presented as the mean value, different letters are significantly different at $\mathrm{P}<0.05$
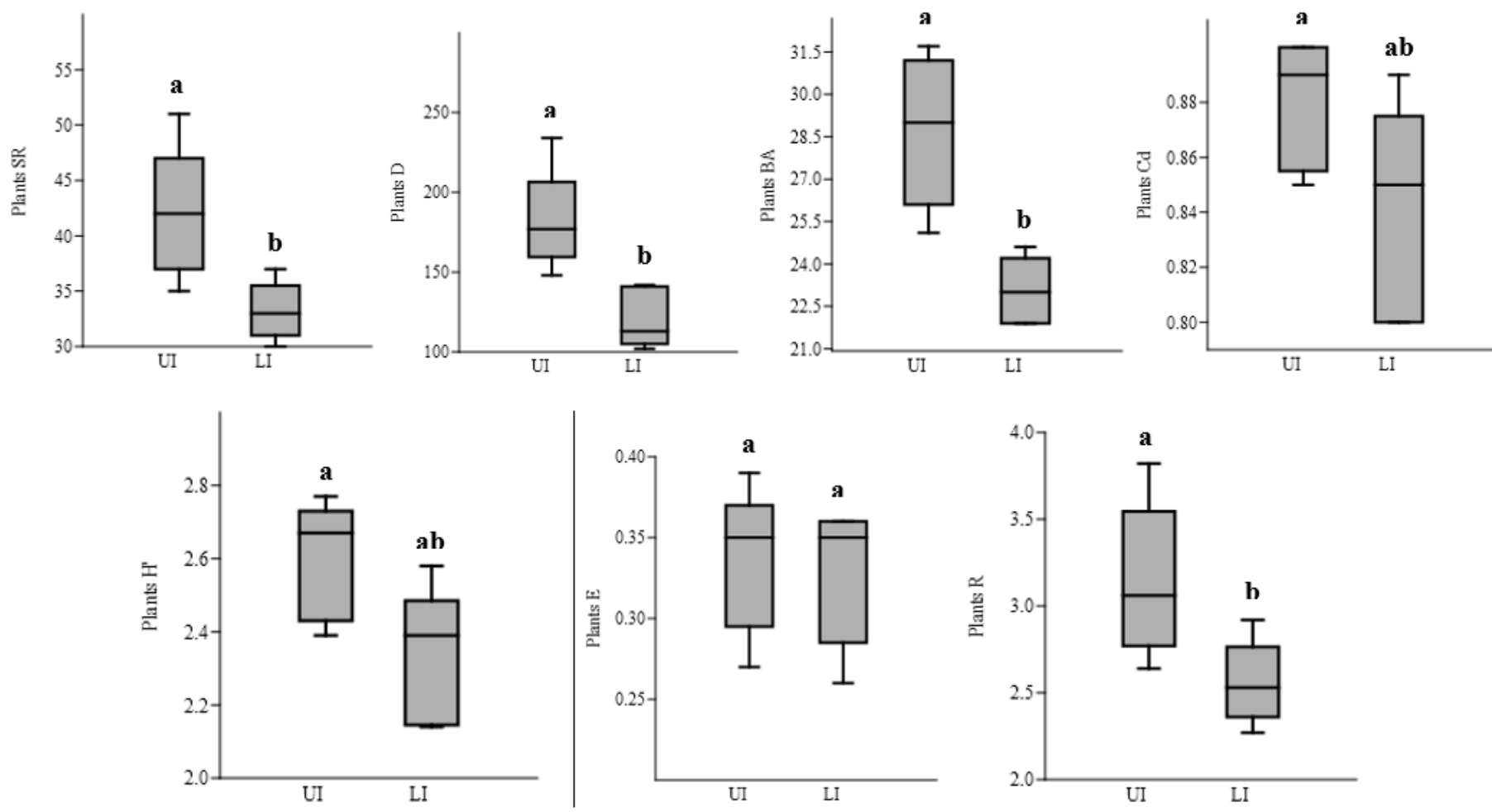

Figure 7

Species richness (SR; No. of species), density (D; stems ha-1), basal area (BA; m2 ha-1), Shannon index (H区), Simpson index (Cd), evenness index (E) and Margalef's index (R) of total plants (trees, herbs and shrubs) in uninvaded (UI) and Lantana-invaded (LI) sites. Data are presented as the mean value, different letters are significantly different at $\mathrm{P}<0.05$

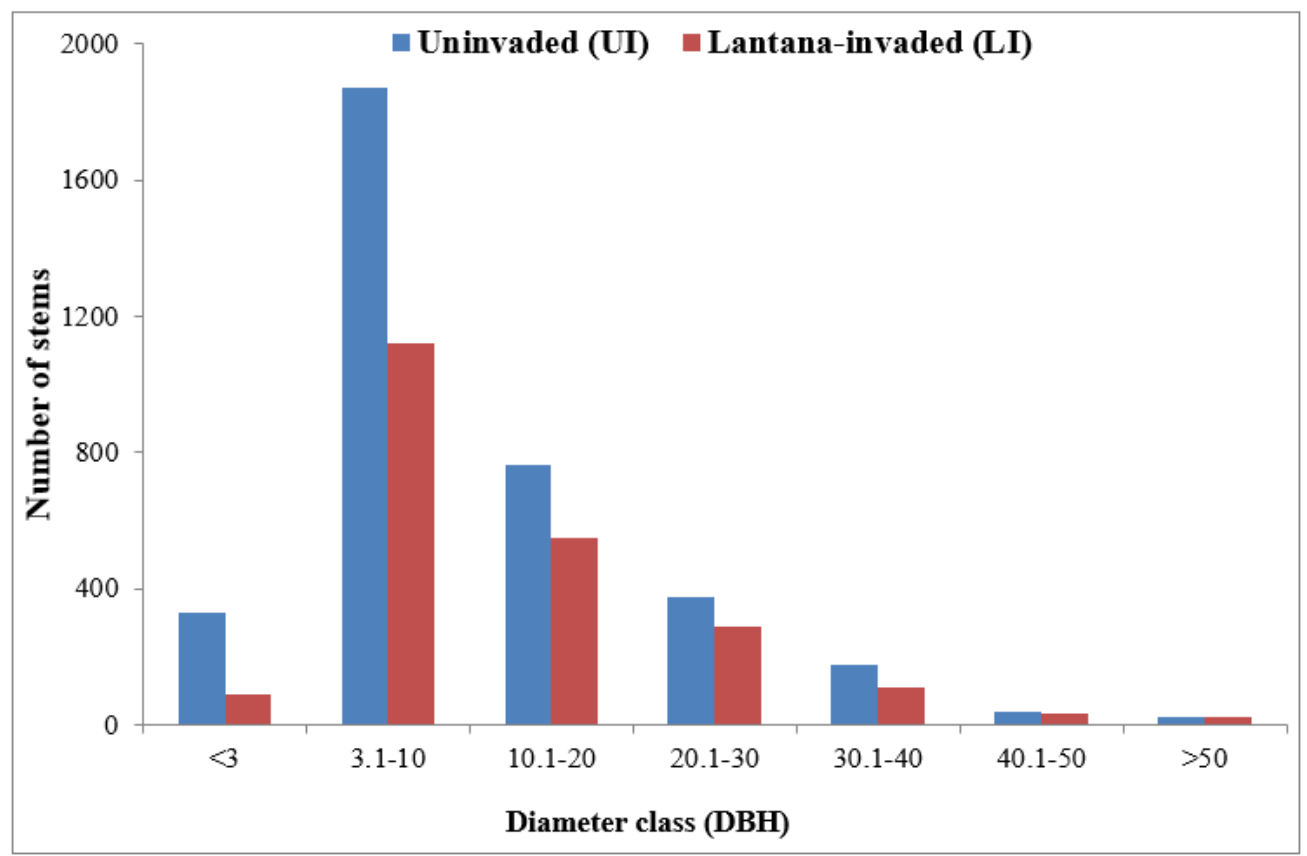

Figure 8

Diameter class-wise distribution of saplings $(<3 \mathrm{~cm})$, juveniles $(>3.1-10 \mathrm{~cm})$ and adult $(>10 \mathrm{~cm})$ trees in terms of stem density in uninvaded $(\mathrm{UI})$ and Lantanainvaded (LI) sites 

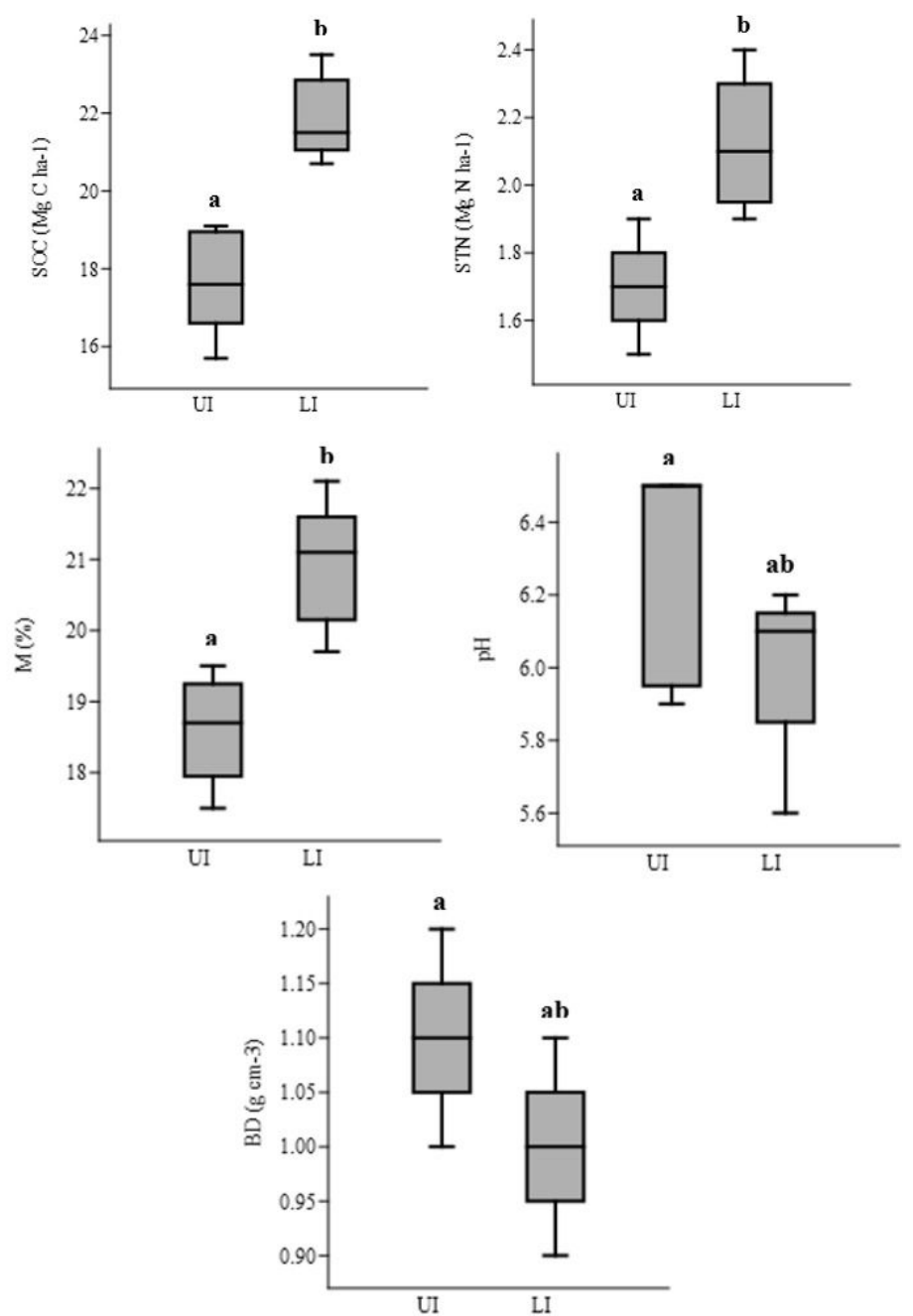

\section{Figure 9}

Soil organic carbon (SOC; Mg C ha-1), soil total nitrogen (STN; Mg C ha-1), moisture (M; \%), pH and bulk density (BD; g cm-3) in uninvaded (UI) and Lantanainvaded (LI) sites. Data are presented as the mean value, different letters are significantly different at $\mathrm{P}<0.05$

\section{Supplementary Files}

This is a list of supplementary files associated with this preprint. Click to download.

- Supplementaryfile1.docx 